\title{
Computation of eigenvalues in proportionally damped viscoelastic structures based on the fixed-point iteration
}

\author{
Mario Lázaro ${ }^{a, *}$, José L. Pérez-Aparicio ${ }^{a}$, Marcelo Epstein ${ }^{\mathrm{b}}$ \\ ${ }^{a}$ Department of Continuum Mechanics and Theory of Structures \\ Universitat Politècnica de València 46022 Valencia, Spain \\ ${ }^{b}$ Department of of Mechanical and Manufacturing Engineering \\ University of Calgary, Calgary, Canada
}

\begin{abstract}
Linear viscoelastic structures are characterized by dissipative forces that depend on the history of the velocity response via hereditary damping functions. The free motion equation leads to a nonlinear eigenvalue problem characterized by a frequency-dependent damping matrix. In the present paper, a novel and efficient numerical method for the computation of the eigenvalues of linear and proportional or lightly nonproportional viscoelastic structures is developed. The central idea is the construction of two complex-valued functions of a complex variable, whose fixed points are precisely the eigenvalues. This important property allows the use of these functions in a fixed-point iterative scheme. With help of some results in Fixed Point Theory, necessary conditions for global and local convergence are provided. It is demonstrated that the speed of convergence is linear and directly depends on the level of induced damping. In addition, under certain conditions the recursive method can also be used for the calculation of non-viscous real eigenvalues. In order to validate the mathematical results, two numerical examples are analyzed, one for single degree-of-freedom systems and another for multiple ones.
\end{abstract}

Keywords: viscoelastic structures, numerical method, complex eigenvalues, real eigenvalues, fixed-point iteration, proportional damping

\section{Introduction}

Materials of viscoelastic nature are widely used for engineering applications such as vibration isolation or as devices to mitigate earthquake effects in buildings. In order to predict the behavior of such structures, the models must reproduce the response as accurately as possible. In the most general case, the structures that include viscoelastic materials are characterized by hereditary energy dissipation mechanisms: the damping forces depend on the history of the velocity response. Mathematically, this fact is represented by convolution integrals that involve the velocities of the degrees-of-freedom (dof) over certain kernel functions. In general, the dof response $\mathbf{u}(t) \in \mathbb{R}^{q}$ is governed by the following system of linear integro-differential equations

$$
\mathbf{M} \ddot{\mathbf{u}}+\int_{-\infty}^{t} \mathcal{G}(t-\tau) \dot{\mathbf{u}} \mathrm{d} \tau+\mathbf{K u}=\mathbf{F}(t)
$$

where $\mathbf{M} \in \mathbb{R}^{q \times q}$ and $\mathbf{K} \in \mathbb{R}^{q \times q}$ are the mass and stiffness matrices assembled using the finite element method. We assume $\mathbf{M}$ to be positive definite and $\mathbf{K}$ positive semidefinite; $\mathcal{G}(t) \in \mathbb{R}^{q \times q}$ is the viscoelastic

\footnotetext{
${ }^{*}$ Corresponding author. Tel +34 963877000 (Ext. 76732). Fax +34 963877189

Email addresses: malana@mes.upv.es (Mario Lázaro), jopeap@mes.upv.es (José L. Pérez-Aparicio), mepstein@ucalgary.ca (Marcelo Epstein)
} 
damping matrix in the time domain.

Checking solutions with form $\mathbf{u}(t)=\mathbf{u}_{0} e^{s t}$ in the free-motion equation (1) with $\mathbf{F}(t) \equiv \mathbf{0}$, the following nonlinear eigenvalue problem is obtained

$$
\left[s^{2} \mathbf{M}+s \mathbf{G}(s)+\mathbf{K}\right] \mathbf{u}_{0}=\mathbf{0}
$$

where $\boldsymbol{G}(s)=\mathcal{L}\{\mathcal{G}(t)\}$ is the damping matrix in the Laplace domain. Many real structures modeled by Eq. (1) present a proportional — or lightly non-proportional- damping matrix, that is, $\mathbf{G}(s)$ becomes diagonal - or diagonally-dominant - in the modal space of the undamped problem. The diagonalization greatly simplifies the calculation of the eigenvalues, since the characteristic equation can be approximated as a product of $q$ decoupled modal equations. The knowledge of the eigensolutions of the previous problem is a key issue in the analysis and study of viscoelastic structures, hence the importance of the development of efficient tools oriented to their numerical computation.

The seminal work of Biot [1] was a starting point in the variational justification of hereditary constitutive models based on exponential decay. Subsequently, experimental results led to extend these models with the introduction of the fractional derivative as a highly effective tool. Papers of Bagley and Torvik [2, 3] established the theoretical basis for the application of fractional derivatives in structural dynamics. Since then, a great number of papers have studied the resulting differential equations in fractional derivatives; among others [4] the works of Ray et al. [5, 6, 7] are of special interest. Both Biot's and fractional derivative models are associated with a different damping function $\mathcal{G}(t)$. The conditions under which this function defines a strictly dissipative motion were studied by Golla and Hughes [8]. Thus, new models could be introduced provided that they satisfy the requirements from [8]. For instance, Buhariwala [9, 10] generalized Biot's model with another in which the relaxation parameters are distributed in an interval. Of special interest are models based on the state-space approach such as the GHM approach of Golla, Hughes and McTavish [8, 11] or the Anelastic Displacement Field of Lesieutre and Mingori [12]. Both of them are characterized by the introduction of new internal variables. Adhikari and Woodhouse [13, 14] proposed new viscoelastic functions in the context of viscous and non-viscous damping identification for dynamic systems. These viscoelastic functions are the most used in the field of structural dynamics and all of them are strictly decreasing in the time domain. However, kernels not necessarily decreasing can also be compatible with viscoelastic problems as it has been demonstrated in the works of Medjden and Tatar [15] and Tatar [16].

Since the linearity is preserved in viscoelastic structures, it is logical to consider the associated eigenvalue problem. The main difference between the models of these systems and those of viscous ones is that for the former the damping matrix is frequency-dependent, see Eq (2), and that the eigenvalue problem is nonlinear. Among the numerical methods oriented to solve this problem, we mention first the works of Yang [17] and Singh [18], developed for any form of the transcendental matrix — named dynamic stiffness matrix in our context-. Both of them are based on the widely used Newton-Raphson method; although the iterative scheme is locally convergent with quadratic speed, the effectiveness depends on the chosen starting point. Another method from Williams and Kennedy [19] suggested a parabolic interpolation of the determinant of the dynamic stiffness matrix. Asymptotic techniques based on perturbation of the damping matrix were proposed by Daya and Potier-Ferry [20] and by Duigou et al. [21]. Voss [22, 23] introduced two methods based on the Arnoldi's shift-and-invert technique and on the Jacobi-Davidson method, respectively. Abdel-Aziz and El-Sayed [24] studied the sensitivity analysis of the eigenvalue functions of non-linear eigensystems under the assumption that such functions are continuous nondifferentiable. Specific methods aimed at solving the problem when the damping function is rational can be found. For instance, Muravyov and Menon [25, 26, 27] developed approaches that essentially transform the nonlinear problem into a larger linear one. Although their solution is exact, the numerical complexity increases with the polynomial degree of the damping function denominator. For proportional or lightly non-proportional systems, Adhikari and Pascual $[28,29]$ proposed several iterative methods based on Taylor series expansion of the damping function to compute complex and real eigenvalues. Friswell and Adhikari [30] proposed a new non-local viscoelastic 
foundation model for beams and obtained the eigensolutions using the finite element method. The introduction of eigenvalues' derivatives in viscoelastic structures [31, 32] has allowed the development of new numerical methods for the computation of the eigensolutions. Cortés and Elejebarrieta [33, 34] developed an approach using the eigenvalue sensitivities, even applicable for highly damped systems. Martinez-Aguirre and Elejebarrieta [35] used higher order eigensensitivities to propose a new numerical procedure for the modal analysis of viscoelastic damped structures. Lázaro and Pérez-Aparicio [36, 37] have carried out recently new proposals based on considering a parametric treatment of the eigensolutions; in them the eigenvalue problem is transformed into an ordinary differential equation.

The present paper develops a new numerical method to compute the eigenvalues of linear viscoelastic structures with proportional — or lightly non-proportional - damping. The key idea is to build two complexvalued functions of a complex variable, whose fixed points are the eigenvalues. These functions uniquely depend on the damping and do not require the calculation of derivatives for their construction. It is shown that the use of the fixed-point iteration always allows to find the complex eigenvalues; moreover, it is demonstrated that the level of damping is directly related to the speed of convergence. Under certain conditions the method can also be applied to the computation of the non-viscous real eigenvalues. Finally, theoretical results are illustrated with two numerical examples. First, a single dof system with an exponential damping model is analyzed; since the overdamped region of this system can be analytically defined, this example allows to relate the level of damping and the speed of convergence. Second, a four-dof system with viscoelastic links is analyzed to validate the method for multiple dof systems with proportional damping.

\section{Single Degree-of-Freedom Systems}

\subsection{Eigenvalue Problem and Recursive Functions}

The theoretical fundamentals of the proposed method are developed for single degree-of-freedom systems (sdof). As mentioned, for non-viscously damped systems the dissipative forces are history-dependent on the velocity of the dof $\dot{u}(t)$ via a kernel function $\mathcal{G}(t)$, a characteristic of the damping model. The sdof free motion equation is the counterpart of Eq. (1)

$$
m \ddot{u}+\int_{-\infty}^{t} \mathcal{G}(t-\tau) \dot{u} \mathrm{~d} \tau+k u=0
$$

where $m$ and $k$ are the mass and the linear stiffness associated with the sdof, respectively. Checking solutions with the form $u(t)=u_{0} e^{s t}$, the previous equation is transformed into the following in terms of the variable $s$

$$
m s^{2}+s G(s)+k=0
$$

where again $G(s)=\mathcal{L}\{\mathcal{G}(t)\}$ is the viscoelastic damping function. Golla and Hughes [8] gave the necessary conditions on $G(s)$ to define a strictly dissipative viscoelastic behavior. Several authors [38, 39] have shown that the characteristic Eq. (4) has $2+p(p \geq 0)$ eigenvalues with form $\left\{\lambda, \lambda^{*}, \sigma_{1}, \ldots, \sigma_{p}\right\}$. The roots $\lambda, \lambda^{*}$ are a pair of complex-conjugate numbers associated with exponential-decay oscillatory modes. The rest $\sigma_{r}, 1 \leq r \leq p$, are negative real numbers named non-oscillatory or non-viscous eigenvalues since they are associated with overcritically damped modes, [39].

The eigenvalues of the undamped problem $G(s) \equiv 0$ are $\pm i \omega_{u}$, where $\omega_{u}=\sqrt{k / m}$ is the undamped natural frequency. Introducing the new non-dimensional function

$$
J(s)=\frac{G(s)}{2 m \omega_{u}}
$$

and using $k=m \omega_{u}^{2}$, Eq. (4) can be written as

$$
s^{2}+2 \omega_{u} s J(s)+\omega_{u}^{2}=0
$$


The key issue of the method is to transform this equation so that the resulting structure permits to apply a recursive scheme. For that, let us assume that Eq. (6) may be expressed in the form

$$
s^{2}+2 \omega_{u} s J(s)+\omega_{u}^{2} \equiv[s+A(s)]^{2}+B(s)
$$

There exist infinite solutions for the unknown functions $A(s), B(s)$; in particular one pair can be obtained identifying the coefficients of the terms $s^{0}=1, s^{1}=s$. Thus,

$$
\begin{aligned}
& A(s)=\omega_{u} J(s) \\
& B(s)=\omega_{u}^{2}\left[1-J^{2}(s)\right]
\end{aligned}
$$

Notice that the solutions given in Eq. (8) are not unique, and as will be shown, this proposed solution has important properties. Using the new functions, further changes can be achieved in Eq. (7) to obtain a more suitable expression. Eq. (7) together Eq. (8) produces a difference of two squares, resulting in

$$
\begin{aligned}
s^{2}+2 \omega_{u} s J(s)+\omega_{u}^{2} & =\left[s+\omega_{u} J(s)\right]^{2}+\omega_{u}^{2}\left[1-J^{2}(s)\right] \\
& =\left[s+\omega_{u} J(s)\right]^{2}-\left(i \omega_{u} \sqrt{1-J^{2}(s)}\right)^{2} \\
& =[s-X(s)][s-Y(s)]
\end{aligned}
$$

where $i=\sqrt{-1}$ is the imaginary unity and the complex functions $X(s), Y(s)$ are defined as

$$
X(s)=\omega_{u}\left(-J(s)+i \sqrt{1-J^{2}(s)}\right), \quad Y(s)=\omega_{u}\left(-J(s)-i \sqrt{1-J^{2}(s)}\right)
$$

In the previous definition, $\sqrt{\bullet}$ must be understood as the principal square root of a complex number. Eq. (9) states that, if $a \in \mathbb{C}$ represents any eigenvalue of the viscoelastic system, then either $a=X(a)$ or $a=Y(a)$, that is, $a$ is a fixed point of some of the two functions $X(s)$ or $Y(s)$. The objective is now to provide the conditions under which these functions may be used in a recursive scheme to calculate the eigenvalues. Previously, some definitions are given to present the hypotheses assumed for the damping function $J(s)$. Let us define the sets

$$
\mathcal{C}_{+}=\{x+i y \in \mathbb{C}: y>0\}, \quad \mathcal{C}_{-}=\{x+i y \in \mathbb{C}: y<0\}
$$

The complex plane excluding real numbers is named $\mathcal{C}=\mathcal{C}_{+} \cup \mathcal{C}_{-}=\mathbb{C} \backslash \mathbb{R}$.

As mentioned, we assume that $G(s)=2 m \omega_{u} J(s)$ verifies the required conditions given by [8] to induce a dissipative motion. In addition, the following two hypotheses are required for the purposes of the current work

H1. $G(s)$ is analytical in a domain with form $\mathbb{C} \backslash \mathcal{M}$, where $\mathcal{M} \subset \mathbb{R}$ is a subset of real numbers

H2. $G(s) \in \mathcal{C}, \forall s \in \mathcal{C}$. Expressed in set operations: $G(\mathcal{C}) \subset \mathcal{C}$

The set $\mathcal{M}$ introduced in $\mathrm{H} 1$ may adopt different forms depending on the type of damping function considered. Table 1 shows the most used damping functions from the bibliography and their associated sets $\mathcal{M}$. It can be observed that, although $\mathrm{H} 1$ assumes the most general case $\mathcal{M} \subset \mathbb{R}$, for real systems $\mathcal{M} \subset \mathbb{R}^{-} \cup\{0\}$ in order to guarantee the energy decay during the free motion.

The functions $X(s), Y(s)$ introduced in Eqs. (10) are directly related with the dimensionless damping function $J(s)=G(s) / 2 m \omega_{u}$. In order to define mathematically the region where $X(s)$ and $Y(s)$ are analytical, let us introduce the set

$$
\mathcal{B}=\{x \in \mathbb{R}:|J(x)| \geq 1\}
$$

Assuming that $G(s)$ satisfies $\mathrm{H} 1, \mathrm{H} 2$, the following properties for $X(s), Y(s)$ can be established (see Appendix A for the proof details) 


\begin{tabular}{lll}
\hline Damping functions, $G(s)$ & Set $\mathcal{M}$ & Author, Year, Reference \\
\hline$\sum_{k=1}^{n} \frac{a_{k}}{s+b_{k}}, \quad b_{k}>0$ & $\left\{-b_{k}\right\}_{k=1}^{n}$ & Biot, 1955 [1] \\
$\frac{c}{b-a} \log \frac{s+b}{s+a}, \quad 0<a<b$ & {$[-b,-a]$} & Buhariwala, 1982 [9, 10] \\
$\frac{1}{s} \frac{E_{1} s^{\alpha}-E_{0} b s^{\beta}}{1+b s^{\beta}}, \quad 0<\alpha, \beta<1$ & $\mathbb{R}^{-} \cup\{0\}$ & Bagley and Torvik, 1983 [3] \\
$\frac{G^{\infty}}{s}\left[1+\sum_{k=1}^{n} \alpha_{k} \frac{s^{2}+2 \zeta_{k} \omega_{k} s}{s^{2}+2 \zeta_{k} \omega_{k} s+\omega_{k}^{2}}\right]$ & $\left\{-\omega_{k}\left(\zeta_{k} \pm \sqrt{\zeta_{k}^{2}-1}\right)\right\}_{k=1}^{n} \cup\{0\}$ & Golla and Hughes, 1983 [8] and \\
$\frac{1}{s}\left[1+\sum_{k=1}^{n} \frac{\Delta_{k} s}{s+\beta_{k}}\right], \quad \beta_{k}>0$ & $\left\{-\beta_{k}\right\}_{k=1}^{n} \cup\{0\}$ & McTavish and Hughes, 1993 [11] \\
$c \frac{1-e^{-s t_{0}}}{s t_{0}}$ & $\emptyset$ & Lesieutre and Mingori, 1990 [12] \\
$c e^{s^{2} / 4 \mu}\left[1-\operatorname{erf}\left(\frac{s}{2 \sqrt{\mu}}\right)\right]$ & $\emptyset$ & Adhikari, 1998 [13] \\
\hline
\end{tabular}

Table 1: Damping functions from the bibliography and their corresponding sets $\mathcal{M} \subset \mathbb{R}$

P1. The functions $X(s), Y(s)$ are analytic in the set $\mathcal{A}=\mathbb{C} \backslash(\mathcal{M} \cup \mathcal{B})$

P2. $X^{*}(s)=Y\left(s^{*}\right), \forall s \in \mathcal{A}$

P3. Let $a \in \mathcal{A}$ be a complex number. Then, $a=X(a)$ if and only if $a^{*}=Y\left(a^{*}\right)$

P4. $X(\mathcal{C}) \subset \mathcal{C}_{+}, \quad Y(\mathcal{C}) \subset \mathcal{C}_{-}$

P5. If $J(\mathbb{R}) \subset \mathbb{R}$ then $X(\mathcal{B}) \subset \mathbb{R}, X(\mathbb{R} \backslash \mathcal{B}) \subset \mathcal{C}_{+}$and also $Y(\mathcal{B}) \subset \mathbb{R}, Y(\mathbb{R} \backslash \mathcal{B}) \subset \mathcal{C}_{-}$

The five properties constitute the starting point to analyze the numerical solution of Eq. (4) from a recursive point of view.

\subsection{Complex Eigenvalues}

The properties P1-P5 allow to state that, if $\lambda \in \mathcal{C}_{+}$and $\lambda^{*} \in \mathcal{C}_{-}$are the complex conjugate pair solution of Eq. (4), then $\lambda=X(\lambda), \lambda^{*}=Y\left(\lambda^{*}\right)$. In other words, $\lambda, \lambda^{*}$ are fixed points of functions $X$ and $Y$, respectively. Let us consider two any complex numbers $x_{0} \in \mathcal{C}_{+}, y_{0} \in \mathcal{C}_{-}$and let $\left\{x_{n}\right\},\left\{y_{n}\right\}$ be the recursive sequences of iterates defined for $n \geq 1$ as

$$
x_{n}=X\left(x_{n-1}\right), \quad y_{n}=Y\left(y_{n-1}\right)
$$

The question is whether these sequences converge or not to fixed points, and therefore, to solutions of the nonlinear eigenvalue problem. An affirmative answer to this question would allow us to use Eqs. (13) as an efficient numerical tool to compute the complex eigenvalues. This subsection is aimed to find answers to the following questions:

- Under what conditions the existence of complex fixed points of $X(s), Y(s)$ can be ensured?

- Can a recursive scheme be constructed so that it converges to these fixed points, regardless of the starting point?

- What is the speed of convergence of the recursive scheme? 
The problem of the existence of fixed points will be solved using a known result in Fixed Point Theory due to Goebel, Sekowski and Stachura [40]. For that, it is necessary to define the concept of strict inclusion in sets.

Definition 1 (Strict inclusion). Let $S, T \subset \mathbb{C}$ two complex sets; $S$ is said to lie strictly inside $T$ if there exists a real positive number $\epsilon>0$ such that $B(w, \epsilon) \subset T, \forall w \in S$. Here, $B(w, \epsilon)$ is the closed ball centered at $w$ with radius $\epsilon$

Lemma 1 (Goebel, Sekowski and Stachura [40]). Let $h: \mathbb{D} \rightarrow \mathbb{D}$ be an analytic self-mapping defined in the open unit ball $\mathbb{D}=\{w \in \mathbb{C}:|w|<1\}$. Let $w_{n}=h\left(w_{n-1}\right)$ be the sequence of iterates of function $h(w)$. Then $h$ has a fixed point in $\mathbb{D}$ if and only if there exists a point $w_{0} \in \mathbb{D}$ such that strictly $\left\{w_{n}\right\}_{n>1} \subset \mathbb{D}$

In addition, to answer the second question another result in Fixed Point Theory due to Reich [41] will be applied. This result is focused on the approximation to fixed points of holomorphic functions in the complex unit ball.

Lemma 2 (Reich [41]). Let $h: \mathbb{D} \rightarrow \mathbb{D}$ be an analytic self-mapping with a fixed point, and let $u_{0} \in \mathbb{D}$. Then the sequence $\left\{u_{n}\right\}$ where $0<\theta<1$

$$
u_{n}=\frac{u_{0}}{n^{\theta}}+\left(1-\frac{1}{n^{\theta}}\right) h\left(u_{n-1}\right), \quad n \geq 1
$$

converges strongly to a fixed point of $h(w)$.

Based on these results Theorem 1 states that, under relatively weak conditions, $X(s), Y(s)$ have fixed points in $\mathcal{C}_{+}$and $\mathcal{C}_{-}$, respectively. Furthermore, an approximation sequence to the fixed points can be generated, regardless of the chosen initial point.

Theorem 1. Let $G(s)$ be a damping function that satisfies the hypothesis H1, H2. Let $\left\{x_{n}\right\}$ be the sequence introduced in Eq. (13). Then:

i) The viscoelastic system given by Eq. (3) has a complex eigenvalue $\lambda \in \mathcal{C}_{+}$if and only if there exists an initial $x_{0} \in \mathcal{C}_{+}$such that

$$
\lim _{n \rightarrow \infty} x_{n} \notin \mathbb{R}
$$

ii) Moreover, for all $z_{0} \in \mathcal{C}$, there exists a sequence $\left\{z_{n}\right\} \subset \mathcal{C}_{+}$, with $z_{1}=X\left(z_{0}\right)$ such that

$$
\lim _{n \rightarrow \infty} z_{n}=\lambda
$$

Proof. i) As a stratightforward result of the hypothesis, $\left\{x_{n}\right\}$ can not have accumulation points in $\mathbb{R}$. From property $\mathrm{P} 4, X\left(x_{n}\right) \in \mathcal{C}_{+}$holds, $\forall n \geq 0$ and $\forall x_{0} \in \mathcal{C}_{+}$. Consequently, there exists a real positive number $\epsilon>0$ such that $B\left(x_{n}, \epsilon\right) \subset \mathcal{C}_{+}$, for $n \geq 1$. Hence, it follows that the set $\left\{x_{n}\right\}_{n=1}^{\infty}$ lies strictly inside the set $\mathcal{C}_{+}$.

To apply the Lemma 1, the domain $\mathcal{C}_{+}$can be transformed into the open unit ball by the Cayley transformation $\psi: \mathcal{C}_{+} \rightarrow \mathbb{D}$, defined through the conformal mapping

$$
\psi(s)=\frac{i \omega_{u}-s}{i \omega_{u}+s}
$$

that transforms the upper half complex plane, $\mathcal{C}_{+}$into the open unit ball $\mathbb{D}$. For instance, the point $i \omega_{u}$ is transformed into the origin, and the boundary $\partial \mathcal{C}_{+}=\mathbb{R}$ into the circumference $\partial \mathbb{D}=\{w \in \mathbb{C}:|w|=1\}$. Now, let us define by composition the following complex function

$$
U=\psi \circ X \circ \psi^{-1}: \mathbb{D} \rightarrow \mathbb{D}
$$


where

$$
\psi^{-1}(w)=i \omega_{u} \frac{1-w}{1+w} \quad \in \mathcal{C}_{+} \forall w \in \mathbb{D}
$$

Immediately, $\psi, \psi^{-1}$ are holomorphic in $\mathcal{C}_{+}$and $\mathbb{D}$, respectively. Since $X$ is also holomorphic in $\mathcal{C}_{+} \subset \mathcal{A}$, it follows that $U$ is holomorphic in $\mathbb{D}$. Moreover, from property $\mathrm{P} 3$, it is verified that $X\left(\mathcal{C}_{+}\right) \subset \mathcal{C}_{+}$, and therefore $U(\mathbb{D}) \subset \mathbb{D}$. A sequence $\left\{w_{n}\right\}$ associated to $\left\{x_{n}\right\}$ can be generated, with elements that are images under $\psi$ in $\mathbb{D}$, i.e. $w_{n}=\psi\left(x_{n}\right) \in \mathbb{D}$ for $n \geq 0$. Let us verify that $w_{n}=U\left(w_{n-1}\right)$ holds, using the definition of $U(w)$

$$
w_{n}=\psi\left(x_{n}\right)=\psi\left(X\left(x_{n-1}\right)\right)=(\psi \circ X)\left(x_{n-1}\right)=(U \circ \psi)\left(x_{n-1}\right)=U\left(\psi\left(x_{n-1}\right)\right)=U\left(w_{n-1}\right)
$$

Since the complete sequence $\left\{x_{n}\right\}_{n=1}^{\infty}$ lies strictly inside $\mathcal{C}_{+}$, it is verified $\left\{w_{n}\right\}_{n=1}^{\infty} \subset \mathbb{D}$ strictly. Therefore, from Lemma 1 there exists a fixed point $\gamma \in \mathbb{D}$ of the function $U$, i.e., $U(\gamma)=\gamma$, so that the anti-image $\lambda=\psi^{-1}(\gamma) \in \mathcal{C}_{+}$is a fixed point of $X$. Indeed, using again the definition of $U$

$$
\lambda=\psi^{-1}(\gamma)=\psi^{-1}(U(\gamma))=\left(\psi^{-1} \circ U\right)(\gamma)=\left(\psi^{-1} \circ \psi \circ X \circ \psi^{-1}\right)(\gamma)=X\left(\psi^{-1}(\gamma)\right)=X(\lambda)
$$

Finally, since the viscoelastic problem characterized by $G(s)$ has at most one eigenvalue in $\mathcal{C}_{+}$(see subsection 2.1), $\lambda$ is the unique fixed point of $X$ in $\mathcal{C}_{+}$. From the property P3, $\lambda^{*}=\psi^{-1}\left(\gamma^{*}\right)$ is a fixed point of the function $Y(s)$ and consequently the other complex eigenvalue of the viscoelastic system.

To finish the first part of the proof, let us consider reciprocally that $\lambda \in \mathcal{C}_{+}$is a complex eigenvalue of the viscoelastic system. Therefore, $\gamma=\psi(\lambda) \in \mathbb{D}$ is a fixed point of $U$. Applying Lemma $1,\left\{w_{n}\right\}_{n=1}^{\infty} \subset \mathbb{D}$ strictly and, hence

$$
\lim _{n \rightarrow \infty} x_{n}=\lim _{n \rightarrow \infty} \psi^{-1}\left(w_{n}\right) \notin \mathbb{R}
$$

ii) Assuming now that the system is not overdamped, there exists a unique eigenvalue $\lambda \in \mathcal{C}_{+}$that is fixed point of $X$. As shown in the previous proof, $\gamma=\psi(\lambda)$ is fixed point of the holomorphic self-mapping $U: \mathbb{D} \rightarrow \mathbb{D}$ defined in Eq. (15). Let $z_{0} \in \mathcal{C}$ be any complex number (excluding reals), from property $\mathrm{P} 4$, $z_{1}=X\left(z_{0}\right) \in \mathcal{C}_{+}$and let $u_{1}=\psi\left(z_{1}\right) \in \mathbb{D}$. The following sequence

$$
u_{n}=\frac{u_{1}}{n^{\theta}}+\left(1-\frac{1}{n^{\theta}}\right) U\left(u_{n-1}\right)
$$

for $n \geq 2$ can be constructed in $\mathbb{D}$, where $\theta$ can be any real number in the open interval $0<\theta<1$. From Lemma 2, we can ensure that the sequence $\left\{u_{n}\right\}$ converges to $\gamma$. Consequently the sequence $\left\{z_{n}\right\}$ generated as

$$
z_{n}=\psi^{-1}\left(u_{n}\right)
$$

will converge to $\lambda$.

Theorem 1 allows us to ensure the existence of complex eigenvalues as fixed points of $X(s), Y(s)$. A recursive scheme towards the fixed point can be generated from Eqs. (20), (21), but it is not established whether the sequences of iterates $\left\{x_{n}\right\},\left\{y_{n}\right\}$ from Eq. (13) converge always to eigenvalues. It seems logical to assume that when $\left\{z_{n}\right\}$ converges also does $\left\{x_{n}\right\}$, but it is an unproven conjecture. In fact, if the conditions of Theorem 1 are satisfied, theoretically a fixed point of $X(s)$ could coexist with a non-convergent sequence $\left\{x_{n}\right\}$. In such case, the recursive scheme would lie in an infinite non-convergent loop contained strictly inside $\mathbb{D}$. However, the authors have not found any case with this behavior. Furthermore, for all analyzed cases the sequence $\left\{x_{n}\right\}$ has converged successfully to the complex eigenvalue for any considered initial point $x_{0} \in \mathcal{C}_{+}$. An explicit proof for this convergence is not currently available when the requirements of the function $J(s)$ are just the hypothesis $\mathrm{H} 1, \mathrm{H} 2$. 
Despite this formal lack of proof and motivated by a justification for the efficiency of the numerical scheme $\left\{x_{n}\right\}$, new additional conditions on the function $J(s)$ are going to be introduced. First, the following Theorem 2 proves local convergence to fixed points of $X(s), Y(s)$. Second, Theorem 3 establishes the necessary conditions to ensure global convergence of sequences $\left\{x_{n}\right\},\left\{y_{n}\right\}$ to fixed points in a closed ball centered at the undamped eigenvalues $\pm i \omega_{u}$, respectively. Moreover, these theorems will allow us to predict the speed of convergence, aspect not possible just with Theorem 1 . The statement and proofs of these theorems are presented for the function $X(s)$. The application for $Y(s)$ can be obtained as a direct consequence of properties P1 to P5.

Theorem 2. Let $\lambda \in \mathcal{C}_{+}$be the complex eigenvalue of the viscoelastic system (3). If

$$
\left|\frac{\partial J(\lambda)}{\partial s}\right|<\left|\frac{1}{\omega_{u}}+\frac{J(\lambda)}{\lambda}\right|
$$

then there exist two positive real numbers $\delta>0,0<\rho<1$, such that the sequence $\left\{x_{n}\right\}$ converges to $\lambda$ for any initial point $x_{0} \in B(\lambda, \delta)=\{s \in \mathbb{C}:|s-\lambda| \leq \delta\}$. Furthermore,

$$
\left|x_{n}-\lambda\right| \leq \frac{\rho^{n}}{1-\rho}\left|X\left(x_{0}\right)-x_{0}\right|
$$

Proof. The proof is based on verification of the function $X(s)$ satisfying Banach's contraction mapping principle $[42,43]$. As well known, this principle constitutes one of the most important results in mathematical analysis. Applied to the complex domain, it states that every contraction self-mapping on a closed set has a unique fixed point. Moreover, the principle allows the bounding of the computed error after $n$ iterations. The proof of this theorem can be organized into two steps:

i) $X(s)$ is contractive in the closed ball $B(\lambda, \delta)$. The derivative of $X(s)$ from Eqs. (10) is

$$
\frac{\partial X}{\partial s}=-\frac{\partial J}{\partial s}\left(1+\frac{i J(s)}{\sqrt{1-J^{2}(s)}}\right) \omega_{u}
$$

Using the inequality given by Eq. $(22)$ and $X(\lambda)=\lambda$

$$
\begin{aligned}
\left|\frac{\partial X(\lambda)}{\partial s}\right| & =\left|\frac{\partial J(\lambda)}{\partial s}\right|\left|1+\frac{i J(\lambda)}{\sqrt{1-J^{2}(\lambda)}}\right| \omega_{u}=\left|\frac{\partial J(\lambda)}{\partial s}\right|\left|\frac{-J(\lambda)+i \sqrt{1-J^{2}(\lambda)}}{i \sqrt{1-J^{2}(\lambda)}}\right| \omega_{u} \\
& =\left|\frac{\partial J(\lambda)}{\partial s}\right|\left|\frac{X(\lambda)}{\frac{X(\lambda)}{\omega_{u}}+J(\lambda)}\right|=\left|\frac{\partial J(\lambda)}{\partial s}\right|\left|\frac{\lambda}{\frac{\lambda}{\omega_{u}}+J(\lambda)}\right|=\frac{\left|\frac{\partial J(\lambda)}{\partial s}\right|}{\left|\frac{1}{\omega_{u}}+\frac{J(\lambda)}{\lambda}\right|}<1
\end{aligned}
$$

Since $X(s)$ is analytical at $s=\lambda \in \mathcal{C}_{+}$, the absolute value of its derivative, $|\partial X / \partial s| \equiv\left|X^{\prime}(s)\right|$ is continuous in a neighborhood of $\lambda$. Then, there exist a positive real number $\delta>0$ so that the maximum value in $B(\lambda, \delta)$ is

$$
\rho=\max _{|s-\lambda| \leq \delta}\left|\frac{\partial X(s)}{\partial s}\right|<1
$$

In real analysis, the previous conclusion is sufficient to prove that $X(s)$ is contractive by straight application of the mean value theorem. In complex analysis this theorem can not be applied in the same terms, but an analog for analytical complex functions was demonstrated by McLeod [44]: let $u, v \in B(\lambda, \delta)$ be two any complex numbers. Since the ball is obviously a convex set, the complex segment between them is 
$[u, v]=\{\xi u+(1-\xi) v: 0 \leq \xi \leq 1\} \subset B(\lambda, \delta)$. According to the reference [44], there exists a real number $0 \leq \xi_{0} \leq 1$ and two complex numbers $s_{1}, s_{2} \in[u, v]$ such that

$$
X(u)-X(v)=(u-v)\left[\xi_{0} \frac{\partial X\left(s_{1}\right)}{\partial s}+\left(1-\xi_{0}\right) \frac{\partial X\left(s_{2}\right)}{\partial s}\right]
$$

Consequently, taking the absolute value of Eq. (27) and using the bound given by Eq. (26), the Lipschitz continuity of $X(s)$ can be proved by

$$
\begin{aligned}
|X(u)-X(v)| & =\left|(u-v)\left[h \frac{\partial X\left(s_{1}\right)}{\partial s}+(1-h) \frac{\partial X\left(s_{2}\right)}{\partial s}\right]\right| \\
& \leq|u-v|\left[h\left|\frac{\partial X\left(s_{1}\right)}{\partial s}\right|+(1-h)\left|\frac{\partial X\left(s_{2}\right)}{\partial s}\right|\right] \\
& \leq[h \rho+(1-h) \rho]|u-v|=\rho|u-v|
\end{aligned}
$$

Since the Lipschitz coefficient verifies $\rho<1$, the function $X(s)$ is contractive.

ii) $X(s)$ is a self-mapping if given any $s \in B(\lambda, \delta)$ i.e. $|s-\lambda| \leq \delta$, its image $X(s)$ also lies inside the ball, i.e. $X(s) \in B(\lambda, \delta)$ or equivalently $|X(s)-\lambda| \leq \delta$. The previous inequality can be proved just using the contraction property in $B(\lambda, \delta)$

$$
|X(s)-\lambda|=|X(s)-X(\lambda)| \leq \rho|s-\lambda| \leq \rho \delta<\delta
$$

Therefore, the hypotheses from Banach's fixed point theorem, see [43], are satisfied and the convergence of the sequence $\left\{x_{n}\right\}$ from Eq. (13) to the (unique) fixed point of $X(s)$ in the ball $B(\lambda, \delta)$ is ensured. Furthermore, the error decay rate in each iteration can be bounded by

$$
\left|x_{n}-\lambda\right| \leq \frac{\rho^{n}}{1-\rho}\left|X\left(x_{0}\right)-x_{0}\right|, \quad \forall x_{0} \in B(\lambda, \delta)
$$

In view of the previous results, it can be demonstrated, see [45], that the speed of convergence of a fixed point iteration scheme is asymptotically linear. In fact, it is verified that

$$
\lim _{n \rightarrow \infty} \frac{\left|x_{n+1}-\lambda\right|}{\left|x_{n}-\lambda\right|}=\rho<1
$$

Notice that the hypotheses of Theorem 2 are expressed in terms of the unknown eigenvalue $\lambda$. Therefore, there is no information a priori on the error rate $\left|x_{n}-\lambda\right|$ since $\rho$ depends on the unknown $\left|X^{\prime}(\lambda)\right|$. In order to improve this theorem, the subsequent Theorem 3 demonstrates the convergence of $\left\{x_{n}\right\}$ using again Banach's contraction principle, but now in a closed ball centered in the undamped eigenvalue $i \omega_{u}$. In addition, this theorem will allow us to relate the speed of convergence with the damping model characteristics, giving a physical insight into the mathematical results. For that, let us introduce two preliminary definitions: given a positive real number $r>0$, and let $\mathcal{H}_{r}=\left\{s \in \mathbb{C}:\left|s-i \omega_{u}\right| \leq r\right\}$ be the closed ball centered in $i \omega_{u}$ with radius $r$. The following numbers are bounds defined as

$$
\alpha_{r}=\max _{s \in \mathcal{H}_{r}}|J(s)|, \quad \beta_{r}=\omega_{u} \max _{s \in \mathcal{H}_{r}}\left|\frac{\partial J}{\partial s}\right|
$$

The existence of $\alpha_{r}, \beta_{r}$ is guaranteed provided that $\mathcal{H}_{r}$ lies inside the analyticity domain $\mathbb{C} \backslash \mathcal{M}$ of $J(s)$. The presence of $\mathcal{H}_{r}$ allows us to express the necessary conditions of Theorem 3 in terms of $\alpha_{r}, \beta_{r}$. 
Theorem 3. Let $\alpha_{r}, \beta_{r}$ be the bounds defined by Eq. (32). If

$$
\alpha_{r}<1, \quad \beta_{r}\left(1+\frac{\alpha_{r}}{\sqrt{1-\alpha_{r}^{2}}}\right) \equiv \kappa_{r}<1, \quad \frac{\left|X\left(i \omega_{u}\right)-i \omega_{u}\right|}{r\left(1-\kappa_{r}\right)} \leq 1
$$

then there exists an unique fixed point $\lambda$ of $X(s)$ in the set $\mathcal{H}_{r}$ and the rate of convergence of the sequence $x_{n}=X\left(x_{n-1}\right)$ is

$$
\left|x_{n}-\lambda\right| \leq \frac{\kappa_{r}^{n}}{1-\kappa_{r}}\left|X\left(x_{0}\right)-x_{0}\right|, \quad \forall x_{0} \in \mathcal{H}_{r}
$$

Proof. Since the domain $\mathcal{H}_{r} \subset \mathbb{C}$ is a closed set in the complex domain, it is a complete set. To apply again Banach's contraction principle to the function $X: \mathcal{H}_{r} \rightarrow \mathbb{C}$, the proof is again organized in two steps.

i) The function $X(s)$ is contractive in the set $\mathcal{H}_{r}$. From Eq. (32), (34) left, $|J(s)| \leq \alpha_{r}<1$ for any $s \in \mathcal{H}_{r}$. Consequently, the ball $\mathcal{H}_{r}$ lies inside the region where $X(s)$ is analytic, that is, $\mathcal{H}_{r} \subset \mathcal{A}=\mathbb{C} \backslash(\mathcal{M} \cup \mathcal{B})$. The contractivity of $X(s)$ can be proved as in Theorem 2, thus, it is only required that the bound of $\left|X^{\prime}(s)\right|$ is strictly less than one in $\mathcal{H}_{r}$. Using the definitions from Eq. (32) and Eq. (24) we have $\forall s \in \mathcal{H}_{r}$

$$
\begin{aligned}
\left|\frac{\partial X}{\partial s}\right| & =\omega_{u}\left|\frac{\partial J}{\partial s}\right|\left|1+\frac{i J(s)}{\sqrt{1-J^{2}(s)}}\right| \leq \beta_{r}\left(1+\left|\frac{i J(s)}{\sqrt{1-J^{2}(s)}}\right|\right) \\
& =\beta_{r}\left(1+|J(s)|\left|\sum_{k=0}^{\infty}\left(\begin{array}{c}
-1 / 2 \\
k
\end{array}\right)(-1)^{k} J^{2 k}(s)\right|\right) \leq \beta_{r}\left(1+\alpha_{r} \sum_{k=0}^{\infty}\left|\left(\begin{array}{c}
-1 / 2 \\
k
\end{array}\right)\right||J(s)|^{2 k}\right) \\
& \leq \beta_{r}\left(1+\alpha_{r} \sum_{k=0}^{\infty}\left(\begin{array}{c}
-1 / 2 \\
k
\end{array}\right)(-1)^{k} \alpha_{r}^{2 k}\right)=\beta_{r}\left(1+\frac{\alpha_{r}}{\sqrt{1-\alpha_{r}^{2}}}\right)=\kappa_{r}<1
\end{aligned}
$$

where the last inequality holds from Eq. (33) middle. In the previous development, $\alpha_{r}<1$ has been used to express the bound of $1 / \sqrt{1-J^{2}(s)}$ in terms of $\alpha_{r}$ through its series expansion. It can be concluded the complex function $X(s)$ is contractive in the set $\mathcal{H}_{r}$.

ii) The function $X(s)$ is a self-mapping of the set $\mathcal{H}_{r}$, i.e., $X\left(\mathcal{H}_{r}\right) \subset \mathcal{H}_{r}$. We focus now on the verification of $X(s) \in \mathcal{H}_{r}, \forall s \in \mathcal{H}_{r}$. Thus, let us consider a $s \in \mathbb{C}$ such that $\left|s-i \omega_{u}\right| \leq r$, hence, $X(s) \in \mathcal{H}_{r}$ holds if $\left|X(s)-i \omega_{u}\right| \leq r$. Using the hypothesis from Eq. (33) right, it follows

$$
\begin{aligned}
\left|X(s)-i \omega_{u}\right| & =\left|X(s)-X\left(i \omega_{u}\right)+X\left(i \omega_{u}\right)-i \omega_{u}\right| \leq\left|X(s)-X\left(i \omega_{u}\right)\right|+\left|X\left(i \omega_{u}\right)-i \omega_{u}\right| \\
& \leq \kappa_{r}\left|s-i \omega_{u}\right|+\left|X\left(i \omega_{u}\right)-i \omega_{u}\right| \leq \kappa_{r} r+\left|X\left(i \omega_{u}\right)-i \omega_{u}\right| \\
& \leq \kappa_{r} r+\left(1-\kappa_{r}\right) r=r
\end{aligned}
$$

In summary, the function $X(s)$ and the ball $\mathcal{H}_{r}$ verifiy the following three properties

- $\mathcal{H}_{r}$ is closed and therefore a complete subset in the complex domain

- $X\left(\mathcal{H}_{r}\right) \subset \mathcal{H}_{r}$

- $X(s)$ is contractive in $\mathcal{H}_{r}$ with Lipschitz coefficient $\kappa_{r}<1$

Therefore, the Banach's contraction principle can to be applied again for $X(s)$, now in $\mathcal{H}_{r}$ assuring the uniqueness of the fixed point $\lambda \in \mathcal{H}_{r}$. Furthermore, the speed of convergence of the sequence $x_{n} \rightarrow \lambda$ is linear and the error can be bounded by

$$
\left|x_{n}-\lambda\right| \leq \frac{\kappa_{r}^{n}}{1-\kappa_{r}}\left|X\left(x_{0}\right)-x_{0}\right|, \quad \forall x_{0} \in \mathcal{H}_{r}
$$


Notice that no reference to the eigenvalue $\lambda$ in the three hypotheses has been made, then, given a radius $r>0$, they can easily be tested a priori provided that $\alpha_{r}, \beta_{r}$ are available. These bounds depend on the mathematical form of the damping function $J(s)$ and they must be searched in the boundary of $\mathcal{H}_{r}$, according to the maximum modulus theorem for analytical complex functions. Assuming now that the necessary conditions of Theorem 3 are satisfied, the mathematical expression of the Lipschitz coefficient

$$
\kappa_{r}=\beta_{r}\left(1+\frac{\alpha_{r}}{\sqrt{1-\alpha_{r}^{2}}}\right)
$$

contains relevant qualitative information. On one hand, as shown in Theorem $3, \kappa_{r}$ controls the convergence speed. On the other, its expression, given by Eq. (38), depends directly on the bounds of the (dimensionless) viscoelastic function $J(s)$ and its derivative $J^{\prime}(s)$. The physical meaning of these values is directly related with the characteristics of the damping model: a) $\alpha_{r}$ is the damping function (in absolute value) maximum in $\mathcal{H}_{r}$. Low values of $J(s)$ are associated with lightly damped systems and $\alpha_{r}$ is a measure of the level of damping; b) $\beta_{r} / \omega_{u}$ directly represents the bound of $J^{\prime}(s)$, derivative directly related with the level of viscoelasticity. Mathematically, a system has small viscoelasticity if the damping function $J(s)$ does not present large variations with respect to $s$ so that $\beta_{r}$ measures the viscoelasticity in $\mathcal{H}_{r}$. A more complete study of the viscoelasticity quantification may be found in the work of Adhikari and Woodhouse [46]. Since the real valued function $\kappa(\alpha, \beta)=\beta\left(1+\alpha / \sqrt{1-\alpha^{2}}\right)$ is always increasing in the range $\beta>0,0<\alpha<1$, it is expected that lightly damped systems with low viscoelasticity will present faster convergence to the fixed points. Hence, the higher the damping level in the system the slower the convergence to the eigenvalue using the proposed recursive method. This affirmation will be validated throughout the numerical examples.

\subsection{Real Eigenvalues}

Assuming that the damping function $G(s)$ induces a dissipative motion, energy loss may result in either a decreasing amplitude oscillatory motion (complex eigenvalues) or a non-oscillatory motion with exponential decay (real negative eigenvalues). For the first, the system is said to be underdamped and for the second overdamped. In the latter no complex number in the set $\mathcal{C}=\mathbb{C} \backslash \mathbb{R}$ exits as fixed point of the functions $X(s), Y(s)$. Therefore, as a corollary of Theorem 1 both recursive sequences will converge to the same real eigenvalue. Starting from a point $x_{0} \in \mathcal{C}_{+}$the elements of $\left\{x_{n}\right\}$ will be contained in the upper half-plane of the complex domain and will converge to a negative number. At the same time, $\left\{y_{n}\right\}$ will also converge to the same eigenvalue but through the lower half-plane starting now in $x_{0}^{*} \in \mathcal{C}_{-}$. However, in underdamped systems non-viscous real eigenvalues coexist with the complex conjugate eigenvalues pair and the question arises whether the recursive functions, $X(s), Y(s)$ are able to converge to some real eigenvalue.

Property $\mathrm{P} 5$ of the recursive functions states that $X(\mathcal{B}) \subset \mathbb{R}$ and $Y(\mathcal{B}) \subset \mathbb{R}$, provided that $J(\mathbb{R}) \subset \mathbb{R}$. Therefore, the functions $X, Y: \mathcal{B} \rightarrow \mathbb{R}$ are well defined and real valued. Obviously, any real eigenvalue must be a fixed point of some of the recursive functions, $X$ or $Y$. Once more, the fixed point theory provides a result due to Schröeder [47,45] giving the necessary conditions for the convergence (local in this case), to a fixed point. This theorem states that the sequence of iterates $t_{n}=X\left(t_{n-1}\right)$ is locally convergent to a fixed point $\sigma \in \mathbb{R}$ if the derivative fulfills $|\partial X(\sigma) / \partial t|<1$. The same conclusion can be established for the function $Y(t)$. The notation $t$ is used for the real independent variable and should not be confused with time, which only appears in Eq. (3). Since $X(t)$ is continuously differentiable in a neighborhood of $\sigma$, there exists an interval centered in this point, $I=[\sigma-\delta, \sigma+\delta]$ such that

$$
\rho=\max _{t \in I}\left|\frac{\partial X(t)}{\partial t}\right|<1
$$

It can be easily proved that any sequence $\left\{t_{n}\right\}_{n=0}^{\infty}$ that starts in the interval $I$ remains in $I$. Thus, the difference between the $n$th term and the fixed point can be bounded by

$$
\left|\sigma-t_{n}\right|=\left|X(\sigma)-X\left(t_{n-1}\right)\right| \leq \rho\left|\sigma-t_{n-1}\right| \leq \cdots \leq \rho^{n}\left|\sigma-t_{0}\right|
$$


which proves the convergence, $\lim _{n \rightarrow \infty} x_{n}=\sigma$. This theorem can be considered as a weak version of the Banach's contraction principle in the real domain and, like Theorem 2, does not provide practical hypotheses due to its dependence on the (unknown) fixed point. However, the numerical examples will show that, under certain conditions, $X(t), Y(t)$ can present low derivative values. Consequently, testing the convergence of the iterative process starting from some point $t_{0} \in \mathbb{R}$, would permit to find non-viscous eigenvalues.

\section{Multiple Degree-of-Freedom Systems}

The main objective of this section is to apply the previously obtained results for single dof systems to multiple ones. Let us name $\mathbf{M} \in \mathbb{R}^{q \times q}, \mathbf{K} \in \mathbb{R}^{q \times q}, \mathbf{u}(t) \in \mathbb{R}^{q}$ to the mass and to the elastic stiffness matrices, and to the vector grouping the dof's. Damping in non-viscous (or viscoelastic) dynamic systems is modeled by dissipative forces that depend on the history of the dof velocities via kernel functions. The equilibrium of the free motion results in a system of integro-differential equations that can be expressed in matrix form as follows

$$
\mathbf{M} \ddot{\mathbf{u}}+\int_{-\infty}^{t} \mathcal{G}(t-\tau) \dot{\mathbf{u}} \mathrm{d} \tau+\mathbf{K u}=\mathbf{0}
$$

where the matrix $\mathcal{G}(t) \in \mathbb{R}^{q \times q}$ contains the viscoelastic kernel functions in time domain. Testing solutions of the form $\mathbf{u}(t)=\mathbf{u}_{0} e^{s t}$, the previous system becomes the nonlinear eigenvalue problem

$$
\left[s^{2} \mathbf{M}+s \mathbf{G}(s)+\mathbf{K}\right] \mathbf{u}_{0}=\mathbf{0}
$$

where $\mathbf{G}(s)=\mathcal{L}\{\mathcal{G}(t)\} \in \mathbb{C}^{q \times q}$. Let $\mathbf{\Phi}=\left[\phi_{1}, \ldots, \phi_{q}\right] \in \mathbb{R}^{q \times q}$ be the matrix grouping the eigenvectors associated with the undamped system defined by matrices $\mathbf{M}, \mathbf{K}$. The classical orthogonal relations can be written as

$$
\boldsymbol{\Phi}^{T} \mathbf{M} \boldsymbol{\Phi}=\tilde{\mathbf{M}}=\operatorname{diag}\left[m_{j}\right], \quad \boldsymbol{\Phi}^{T} \mathbf{K} \boldsymbol{\Phi}=\tilde{\mathbf{K}}=\operatorname{diag}\left[k_{j}\right]
$$

Changing the variable $\mathbf{u}_{0}=\mathbf{\Phi} \mathbf{z}_{0}$ and using the orthogonal relations, Eq. (42) may be expressed as

$$
\left[s^{2} \tilde{\mathbf{M}}+s \boldsymbol{\Gamma}(s)+\tilde{\mathbf{K}}\right] \mathbf{z}_{0}=\mathbf{0}
$$

where $\boldsymbol{\Gamma}(s)=\boldsymbol{\Phi}^{T} \mathbf{G}(s) \boldsymbol{\Phi}$ is the damping matrix in the modal space. In general, this matrix is not diagonal, only non-viscous proportional damping presents this property. The necessary and sufficient conditions for proportional damping in non-viscous systems have been studied by Adhikari [48]. For the current paper purposes, the hypothesis of light non-proportional damping is considered, i.e., the matrix $\boldsymbol{\Gamma}\left(\lambda_{j}\right)$ is diagonally dominant, that is equivalent to assume as true that

$$
\sum_{\substack{l=1 \\ l \neq k}}^{q}\left|\Gamma_{k l}\left(\lambda_{j}\right)\right|<\left|\Gamma_{k k}\left(\lambda_{j}\right)\right|, \quad \forall 1 \leq k \leq q
$$

where $\lambda_{j}$ is the $j$ th eigenvalue. This assumption is commonly assumed in many problems related with non-viscous damping $[49,39,28,29]$ and it allows the approximation of the determinant as the product of the terms of its main diagonal, that is

$$
\operatorname{det}\left[s^{2} \tilde{\mathbf{M}}+s \boldsymbol{\Gamma}(s)+\tilde{\mathbf{K}}\right] \approx \prod_{j=1}^{q}\left(m_{j} s^{2}+s \Gamma_{j j}(s)+k_{j}\right)
$$

Hence, the set of eigenvalues can be obtained from the following $q$ equations

$$
D_{j}(s)=m_{j} s^{2}+s \Gamma_{j j}(s)+k_{j}=0, \quad 1 \leq j \leq q
$$

In general, the number of eigenvalues of an oscillatory viscoelastic (underdamped) system is $2 q+p$, with $p \geq 0$; there are $2 q$ complex eigenvalues formed by $q$ complex conjugate pairs. In addition, the set of 
eigenvalues must be completed with $p$ real and non-viscous roots. This set is formed by negative numbers associated with overcritical modes, of non-oscillatory nature. The total number of non-viscous roots can be written as $p=p_{1}+\cdots+p_{q}$, where $p_{j} \geq 0,1 \leq j \leq q$ is the number of non-viscous roots of the $j$ th characteristic equation.

In order to apply the recursive scheme, let us define the dimensionless damping functions

$$
J_{j}(s)=\frac{\Gamma_{j j}(s)}{2 m_{j} \omega_{j}}, \quad 1 \leq j \leq q
$$

Following the same procedure as before, the $j$ th equation can be expressed as

$$
\frac{D_{j}(s)}{m_{j}}=s^{2}+2 s J_{j}(s) \omega_{j}+\omega_{j}^{2}=\left[s-X_{j}(s)\right]\left[s-Y_{j}(s)\right]
$$

where

$$
X_{j}(s)=\omega_{j}\left(-J_{j}(s)+i \sqrt{1-J_{j}^{2}(s)}\right), \quad Y_{j}(s)=\omega_{j}\left(-J_{j}(s)-i \sqrt{1-J_{j}^{2}(s)}\right)
$$

are the functions used in recursive form to compute the complex eigenvalues. The fixed point theory provides a sequence defined by Eq. (20) contained in the complex unit ball that strongly converges to the fixed point. However, the sequences

$$
x_{n}=X_{j}\left(x_{n-1}\right), \quad y_{n}=Y_{j}\left(y_{n-1}\right), \quad n \geq 1
$$

behave very well and in practice can efficiently be used to obtain the fixed point. As described in the previous section, the speed of convergence depends on damping level and on viscoelasticity. As the starting point of the algorithm, the undamped eigenvalue $i \omega_{j}$ is in general a good choice; however any suitable initial point can be used as long as it belongs to $\mathcal{C}=\mathbb{C} \backslash \mathbb{R}$.

Finally, for the eigenvectors' computation, two procedures can be used [39]. From the approximated eigenvalue obtained with the recursive process described above $\lambda_{j}$, the components of the $j$ th associated eigenvector, say $\mathbf{u}_{j}$, can be computed. First, from the ill-conditioned linear system of equations

$$
\left[\lambda_{j}^{2} \mathbf{M}+\lambda_{j} \mathbf{G}\left(\lambda_{j}\right)+\mathbf{K}\right] \mathbf{u}_{j}=\mathbf{0}
$$

This method can be computationally inefficient for large size systems because it involves the calculation of an inverse submatrix of dimension $q-1$; in any case it is specially recommended for non-viscous eigenvectors associated to non-viscous eigenvalues, for which no alternative method is currently available. Second, for the complex eigenvectors, the hypothesis of non-proportional damping allows the use of approximate expressions. The complex eigenvectors are expanded in terms of the off-diagonal terms of matrix $\boldsymbol{\Gamma}\left(\lambda_{j}\right)=\boldsymbol{\Phi}^{T} \mathbf{G}\left(\lambda_{j}\right) \boldsymbol{\Phi}$. Let us name $\mathcal{D}_{j}(s)=D_{j}(s) / m_{j}$ to the expression given by Eq. (49) and $\boldsymbol{\psi}_{j}=\boldsymbol{\phi}_{j} / m_{j}$ to the mass-normalized $j$ th undamped eigenvector, for $1 \leq j \leq q$. The second order approximation of the $j$ th eigenvector is expressed as linear combination of the undamped eigenvectors $\left\{\boldsymbol{\psi}_{1}, \ldots, \boldsymbol{\psi}_{q}\right\}$, which forms a base of the space $\mathbb{R}^{q}$.

$$
\mathbf{u}_{j} \approx \boldsymbol{\psi}_{j}-\lambda_{j} \sum_{\substack{k=1 \\ k \neq j}}^{q} \frac{\Gamma_{k j}\left(\lambda_{j}\right)}{\mathcal{D}_{k}\left(\lambda_{j}\right)} \boldsymbol{\psi}_{k}+\lambda_{j}^{2} \sum_{\substack{k=1 \\ k \neq j}}^{q} \sum_{\substack{l=1 \\ l \neq j \neq k}}^{q} \frac{\Gamma_{k l}\left(\lambda_{j}\right) \Gamma_{l j}\left(\lambda_{j}\right)}{\mathcal{D}_{k}\left(\lambda_{j}\right) \mathcal{D}_{l}\left(\lambda_{j}\right)} \boldsymbol{\psi}_{k}
$$

\section{Numerical Examples}

\subsection{Example 1: Single Degree-of-Freedom Systems}

In order to validate the theoretical developments a single dof system is analyzed. For that, the Biot's viscoelastic model [1] with one exponential kernel as the hereditary damping function is used.

$$
\mathcal{G}(t)=c_{v} \mu e^{-\mu t}
$$


where $\mu>0$ is the relaxation parameter and $c_{v}$ the damping coefficient of the limit viscous model when $\mu \rightarrow \infty$. Let us denote by $\zeta=c_{v} / 2 m \omega_{u}$ the damping ratio of the limit viscous model, then the Laplace transform of the damping function is

$$
G(s)=2 m \omega_{u} \zeta \frac{\mu}{s+\mu}
$$

The characteristic equation is of the form

$$
s^{2}+2 s \omega_{u} \zeta \frac{\mu}{s+\mu}+\omega_{u}^{2}=0
$$

Multiplying the previous equation by $s+\mu$, it is transformed into a third order polynomial. Introducing now the new variables $z=s / \omega_{u}, \nu=\omega_{u} / \mu$ the following non-dimensional equation is obtained

$$
(\nu z+1)\left(z^{2}+1\right)+2 z \zeta=0
$$

Several authors $[26,50,38,51]$ have studied the dynamics of the described viscoelastic oscillator through the solutions of the previous polynomial. The three roots will be either a complex conjugate pair together with a single real number or three negative real numbers. The so-called overdamped region is defined by the set of damping parameters $\nu, \zeta$ that induce an overdamped motion, i.e. Eq. (57) gives three negative real roots. Adhikari [51] carried out a rigorous analysis of the $\nu, \zeta$ domain. Among other properties, the reference obtained closed expressions of the region boundary in terms of $\nu, \zeta$. In Fig. 1 left the representation of the overdamped (OD) region is drawn as a grey area. The rest (white area) will be the underdamped region (UD).

The availability of an explicitly defined overdamped region has motivated us to adopt Biot's damping model with one kernel. Convergence properties of the proposed recursive method and damping characteristics will be related. By means of straightforward operations, it can be verified that the particular function $J(s)=\zeta \mu /(s+\mu)$ satisfies H1, H2. If the sequence $x_{n}=X\left(x_{n-1}\right)$ does not converge to the set $\mathbb{R}$ for some initial point $x_{0} \in \mathcal{C}_{+}$, then Theorem 1 ensures the existence of a complex fixed point of the function $X(s)$. Furthermore, if the damping model induces an overdamped motion, the sequence converges to a real eigenvalue but the theorem does not give information about the convergence speed. At this regard, Theorems 2 and 3 state that the Banach's contraction principle can be applied under certain conditions. The theoretical results have shown that lightly damped systems with low viscoelasticity present faster convergence to the eigenvalue.

Given a pair $(\zeta, \nu)$ the recursive scheme $x_{n}=X\left(x_{n-1}\right)$ can be carried out to approximate the eigenvalue, starting always from the initial point $x_{0}=i \omega_{u}$. Since $y_{n}=x_{n}^{*}$, the sequence $\left\{y_{n}\right\}$ is not of interest provided that $y_{0}=x_{0}^{*}$. It is considered that the method has reached a solution when the error defined by

$$
\epsilon_{n}=\left|\frac{x_{n}-x_{n-1}}{x_{1}-x_{0}}\right|
$$

is lower than the prefixed value $\epsilon_{\max }=10^{-12}$. If $\lambda(\zeta, \nu)$ is the eigenvalue obtained as the limit of $\left\{x_{n}\right\}$, the density plot of $\left|X^{\prime}(\lambda(\zeta, \nu))\right|$ is drawn in Fig. 1 right for $\omega_{u}=10 \mathrm{rad} / \mathrm{s}$. It should be noted that for all pairs (points in the plot) the sequence is convergent since the absolute value of the derivative is always less than the unity. The lowest values of $\left|X^{\prime}(\lambda(\zeta, \nu))\right|$ are located in the UD area with factor $0<\zeta<1$ and in the OD region. Therefore, it is expected that the recursive method is faster in these zones whereas the derivative values close to one (on the OD contour) are related with very slow convergence. To illustrate the convergence of the different zones, nine different cases associated with points $\mathrm{C} 1$ to $\mathrm{C} 9$ represented in Fig. 1 left.

In Table 2 the approximated eigenvalue $x_{N}\left(N\right.$ is the number of iterations for $\left.\epsilon_{N}<\epsilon_{\max }\right)$ obtained from the recursive sequence are listed for the nine cases in the fourth column. Due to the special relevance of the recursive function derivative $X^{\prime}(s)=\partial X / \partial s$, its absolute value evaluated in $x_{N}$ and in the undamped 

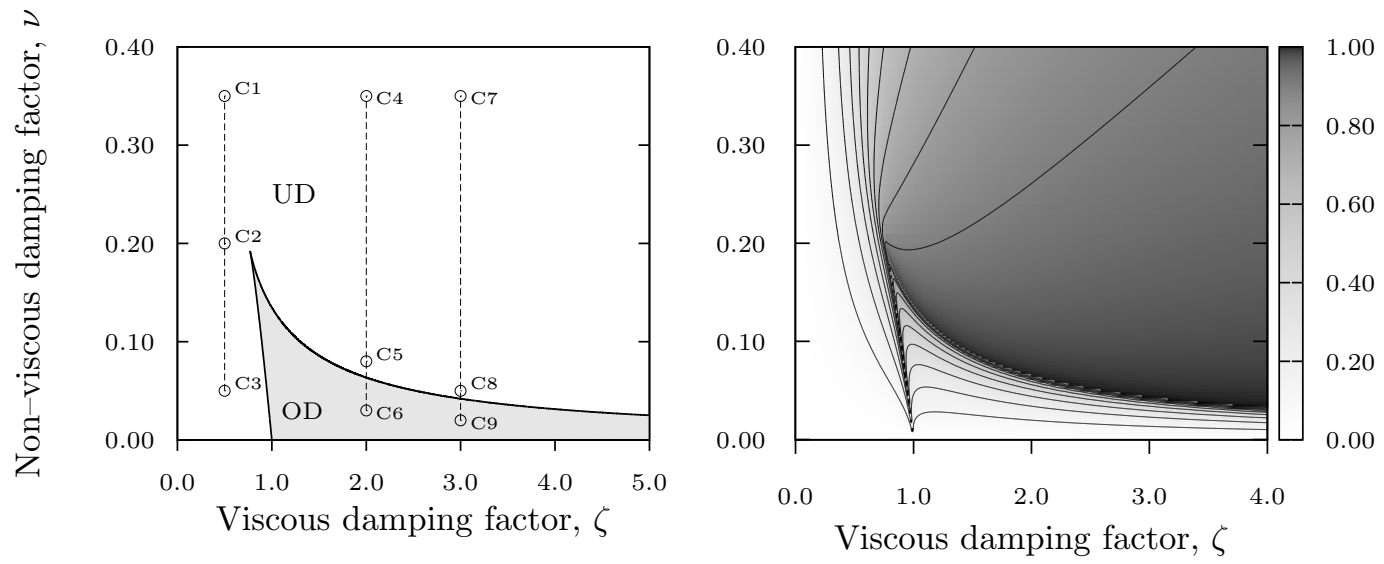

Figure 1: Left: underdamped UD, overdamped OD regions in parametric domain $(\zeta, \nu)$ of Example 1 and points C1 to C9 defined for numerical cases. Right: density plot of $\left|X^{\prime}(\lambda(\zeta, \nu))\right|$ for complex eigenvalue $\lambda(\zeta, \nu)$.

eigenvalue are listed in the last two columns. As expected, C5, C8 require a great number of iterations since the related values of $\left|X^{\prime}(\lambda)\right|$ are close to one. On the contrary, low values of $\left|X^{\prime}(\lambda)\right|$ as for $\mathrm{C} 1, \mathrm{C} 2, \mathrm{C} 3, \mathrm{C} 6$, C9 produce very fast convergence. Points C6, C9 converge to real eigenvalues since they are inside the OD. The number of iterations for $\mathrm{C} 4, \mathrm{C} 7$ is one order of magnitude higher due to the closeness of $\left|X^{\prime}(\lambda)\right|$ to unity.

In Fig. 2, (first nine plots) the complex-domain paths of the computed sequences have been represented together (last three plots) with the error-iterations plots for each numerical case. The initial guess $x_{0}$ is signaled by a circle and the converged eigenvalue by a square. It can be observed that Cases C1to C3 need few iterations to approach a neighborhood of the final eigenvalue. In these cases, the Theorem 3 can be applied and a suitable value of radius $r$ could be found. In the rest of cases, the sequences always converge but the speed of convergence could be predicted only in a neighborhood of the eigenvalue by means of Theorem 2 .

\begin{tabular}{lrrrrrr}
\hline Case & $\zeta$ & $\nu$ & $x_{N}$ & $N$ & $\left|X^{\prime}\left(x_{N}\right)\right|$ & $\left|X^{\prime}\left(i \omega_{u}\right)\right|$ \\
\hline C1 & 0.50 & 0.35 & $-6.49+11.88 i$ & 27 & 0.3309 & 0.2025 \\
C2 & 0.50 & 0.20 & $-6.21+9.72 i$ & 18 & 0.1677 & 0.1212 \\
C3 & 0.50 & 0.05 & $-5.26+8.82 i$ & 10 & 0.0315 & 0.0296 \\
C4 & 2.00 & 0.35 & $-13.07+31.76 i$ & 193 & 0.8619 & 1.3188 \\
C5 & 2.00 & 0.08 & $-61.19+32.18 i$ & 2983 & 0.9907 & 0.3422 \\
C6 & 2.00 & 0.03 & $-43.76+0.00 i$ & 18 & 0.1678 & 0.1291 \\
C7 & 3.00 & 0.35 & $-13.48+39.86 i$ & 278 & 0.9025 & 1.9178 \\
C8 & 3.00 & 0.05 & $-99.15+43.96 i$ & 9800 & 0.9972 & 0.3083 \\
C9 & 3.00 & 0.02 & $-67.97+0.00 i$ & 18 & 0.1643 & 0.1236 \\
\hline
\end{tabular}

Table 2: Example 1: Approximated complex eigenvalues $x_{N}$ from sequence $x_{n}=X\left(x_{n-1}\right)$ with initial point $x_{0}=i \omega_{u}=10 i$. $N$ number of iterations for error $\epsilon_{N}<10^{-12}$.

To complete this example, a few remarks on the application of the method to non-viscous eigenvalues computation will be made. As known, in a context of oscillatory motion a single dof viscoelastic model can have real eigenvalues (non-viscous eigenvalues), as well as a single complex conjugate pair. In subsection 2.3 it has been demonstrated that under certain conditions the sequences $\left\{x_{n}\right\},\left\{y_{n}\right\}$ may converge to some non-viscous eigenvalue. For the current example, to reach this convergence the derivatives need to verify either $\left|X^{\prime}(\sigma)\right|<1$ or $\left|Y^{\prime}(\sigma)\right|<1$, where $\sigma \in \mathbb{R}$ is any non-viscous real eigenvalue. The analytical expression of these derivatives can be calculated, if $X(\sigma)=\sigma$ or $Y(\sigma)=\sigma$ then 

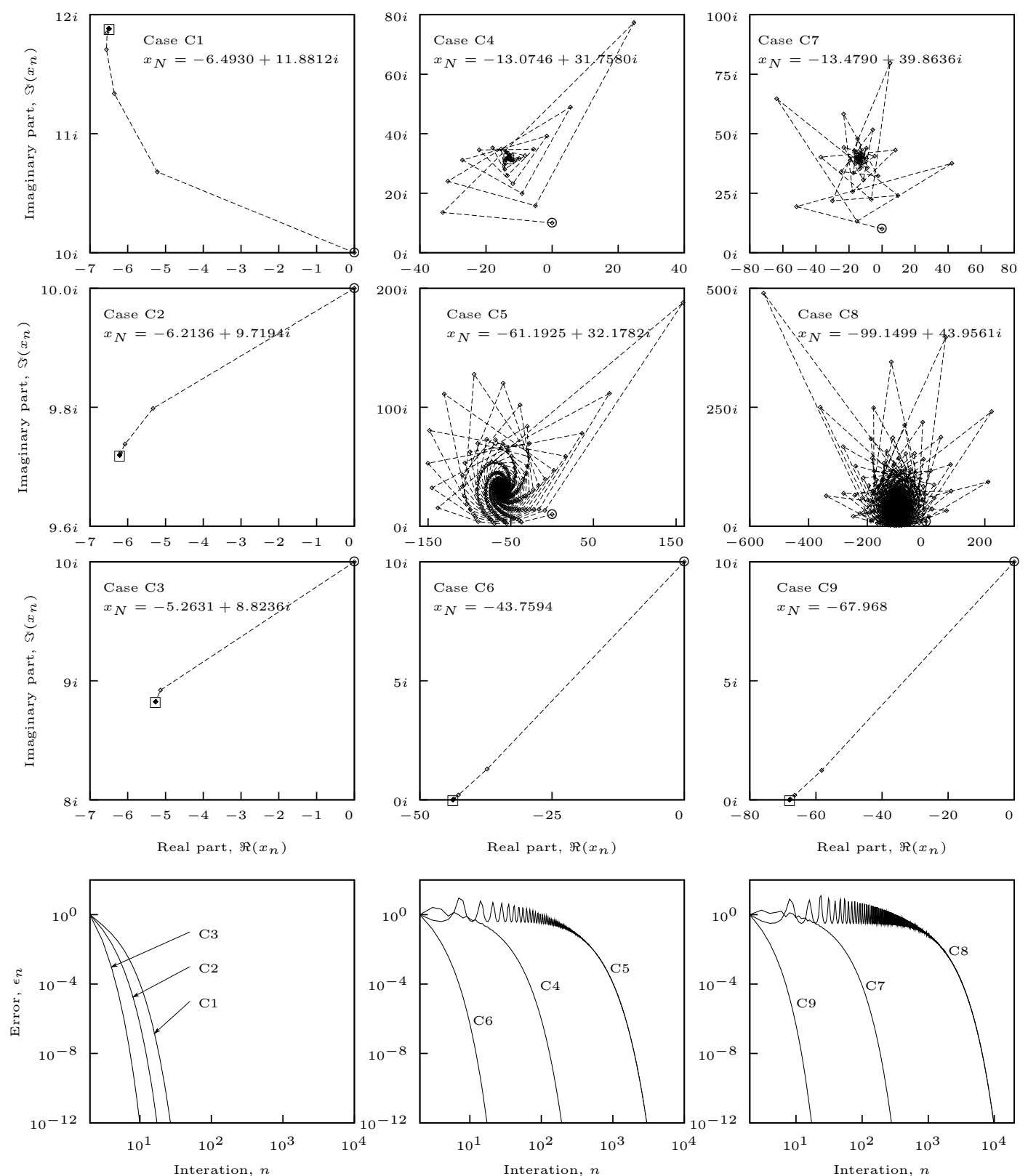

Figure 2: Example 1: Complex path of recursive sequence $x_{n}$ from initial guess $x_{0}=i \omega_{u}=10 i$, point $\odot$, up to the limit eigenvalue $\square$, top nine plots. Associated error-iteration $\epsilon_{n}$ curves, bottom three plots, all for cases in Fig. 1 left. 


$$
X^{\prime}(\sigma)=Y^{\prime}(\sigma)=\frac{\zeta \sigma \mu \omega_{u}}{(\sigma+\mu)\left[\sigma(\sigma+\mu)+\zeta \mu \omega_{u}\right]}
$$

For lightly damped systems, non-viscous eigenvalues $\sigma$ are located close to the pole $-\mu$ of the kernel function, [28]. Then, since the expression $\sigma+\mu \approx 0$ appears in the Eq. (59) denominator, it follows that the lighter the damping the higher the absolute value of the derivatives. For light damping, it is expected that the sequences $\left\{x_{n}\right\},\left\{y_{n}\right\}$ will not converge to real numbers. A high level of damping can facilitate the convergence, resulting in low derivative absolute values that may evenbe less than one. For the current example, the application of the method to obtain non-viscous eigenvalues gives results that are shown in Table 3; the same initial point has been taken for both sequences, i.e., $x_{0}=y_{0}$. The recursive results are considered successful if they converge to a real number with iterations that stay within the real domain, but it can be observed that for all cases the sequence $x_{n}=X\left(x_{n-1}\right)$ does not satisfactorily converge to non-viscous eigenvalues. For example, for cases C1, C2, C3, C7, the sequence $\left\{x_{n}\right\}$ does not stay in the real domain and finally converges to complex eigenvalues. Cases C6, C9 are over-damped and their sequences also escapes from the real numbers line before returning to converge to a real number. The sequences of cases C4, C5, C8 remain inside the real numbers but enter a non convergent infinite loop.

The sequence $y_{n}=Y\left(y_{n-1}\right)$ produces quite different results. Convergence occurs for all high damping cases (C4-C9, in bold) towards non-viscous eigenvalues $\sigma$, verifying $y_{n} \in \mathbb{R}, \forall n \geq 0$. Therefore, the sequence reaches the real numbers remaining in the real axis and obviously $\left|Y^{\prime}(\sigma)\right|<1$. The rest of cases $\mathrm{C} 1$, C2, C3 converge to complex eigenvalues as with $x_{n}=X\left(x_{n-1}\right)$.

\begin{tabular}{lrrrrrrrr}
\hline & & \multicolumn{3}{c}{ Iteration sequence of $X(s)$} & & \multicolumn{3}{c}{ Iteration sequence of $Y(s)$} \\
\cline { 5 - 8 } Case & $x_{0}, y_{0}$ & $x_{N}$ & $N\left|X^{\prime}\left(x_{N}\right)\right|$ & & $y_{N}$ & $N$ & $\left|Y^{\prime}\left(y_{N}\right)\right|$ \\
\hline C1 & -21.43 & $-6.49+11.88 i$ & 29 & 0.3309 & & $-6.49-11.88 i$ & 28 & 0.3309 \\
C2 & -37.50 & $-6.21+9.72 i$ & 26 & 0.1677 & & $-6.21-9.72 i$ & 18 & 0.1677 \\
C3 & -150.00 & $-5.26+8.82 i$ & 11 & 0.0315 & & $-5.26-8.82 i$ & 11 & 0.0315 \\
C4 & -14.29 & - & n.c. & 1.4452 & $\mathbf{- 2 . 4 2 2}$ & 15 & 0.1042 \\
C5 & -62.50 & - & n.c. & 1.0456 & $\mathbf{- 2 . 6 1 5}$ & 10 & 0.0245 \\
C6 & -166.67 & $-43.76+0.00 i$ & 18 & 0.1678 & & $\mathbf{2 . 6 5 5}$ & 8 & 0.0092 \\
C7 & -14.29 & $-13.48+39.86 i$ & 276 & 0.9025 & & $\mathbf{- 1 . 6 1 3}$ & 12 & 0.0631 \\
C8 & -100.00 & - & n.c. & 0.3078 & & $\mathbf{- 1 . 7 0 0}$ & 8 & 0.0091 \\
C9 & -250.00 & $-67.97+0.00 i$ & 18 & 0.1643 & & $\mathbf{- 1 . 7 1 0}$ & 7 & 0.0036 \\
\hline
\end{tabular}

Table 3: Example 1: Results for non-viscous eigenvalues; cases converging to non-viscous eigenvalues emphasized in bold. $N$ number of iterations, $\left|X^{\prime}\left(x_{N}\right)\right|,\left|Y^{\prime}\left(y_{N}\right)\right|$ recursive function derivative evaluated in sequence limit. Non convergent sequences named "n.c."

In this example, it has been shown that the proposed method not only can be used to compute efficiently complex eigenvalues but also non-viscous ones. Furthermore, the convergence to the latter is achieved when the damping induced in the system is high. This duality is an important advantage, because the published methods that are oriented to the calculation non-viscous eigenvalues [28, 29] often assume light damping.

\subsection{Example 2: Multiple Degree-of-Freedom Systems}

To complete the validation of the numerical method, a four-dof discrete system with four masses and two types of viscoelastic links is studied, as shown in Fig. 3. Links $A$ relate internal forces with relative displacements and velocities of consecutive masses (each one an active dof) through a five-kernels Biot's model; links B directly constraint each mass through a four-parameter viscoelastic model based on the 


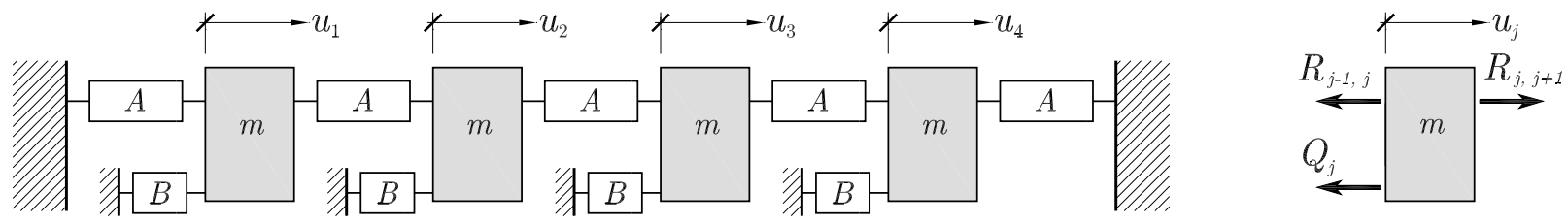

Figure 3: Example 2: Lumped-mass dynamical system with viscoelastic links. Links $A$ follows the Biot's model with five kernels, links $B$ based on the fractional derivatives.

\begin{tabular}{ccccccc} 
Mass $(\mathrm{kg})$ & \multicolumn{2}{c}{ Rigidities $(\mathrm{N} / \mathrm{m})$} & \multicolumn{4}{c}{ Natural frequencies $(\mathrm{rad} / \mathrm{s})$} \\
\hline$m$ & $k_{a}$ & $k_{b}$ & $\omega_{1}$ & $\omega_{2}$ & $\omega_{3}$ & $\omega_{4}$ \\
\hline $10^{3}$ & $10^{5}$ & $5 \times 10^{4}$ & 9.391 & 13.718 & 17.658 & 20.293 \\
\hline
\end{tabular}

Table 4: Example 2: Mass, static rigidities and modal natural frequencies for the 4-dof undamped system.

fractional derivatives [52]. Indexes $j=0,5$ of fixed boundaries are related with zero dof's so that $u_{0}=\dot{u}_{0}=$ $u_{5}=\dot{u}_{5}=0$. Thus, the constitutive equations relating reactions and displacements are

$$
\begin{array}{ll}
\text { Links } A, 1 \leq j \leq 5: & R_{j-1, j}=k_{a}\left(u_{j}-u_{j-1}\right)+\int_{-\infty}^{t} \mathcal{G}_{a}(t-\tau)\left(\dot{u}_{j}-\dot{u}_{j-1}\right) \mathrm{d} \tau \\
\text { Links } B, 1 \leq j \leq 4: & Q_{j}+T_{r}^{\gamma} \frac{\mathrm{d}^{\gamma} Q_{j}}{\mathrm{~d} t^{\gamma}}=k_{b}\left(u_{j}+c T_{r}^{\gamma} \frac{\mathrm{d}^{\gamma} u_{j}}{\mathrm{~d} t^{\gamma}}\right)
\end{array}
$$

where $c, \gamma$ and $T_{r}$ are the parameters of the damping model based on the fractional derivatives and for real materials $c>1,0<\gamma<1, T_{r}>0$. The coefficients $k_{a}, k_{b}$ are the linear, static rigidities of links $A$ and $B$, respectively and the function

$$
\mathcal{G}_{a}(t)=\frac{c_{v}}{5} \sum_{l=1}^{5} \mu_{l} e^{-\mu_{l} t}
$$

is the kernel that controls the damping behavior of links $A$. The damping coefficient $c_{v}=2 m \omega_{a} \zeta$ is expressed as function of a certain damping ratio $\zeta$ and of the reference frequency, $\omega_{a}=\sqrt{k_{a} / m}$. Its Laplace transform is directly

$$
G_{a}(s)=\frac{c_{v}}{5} \sum_{l=1}^{5} \frac{\mu_{l}}{s+\mu_{l}}
$$

resulting for links $A$ the following frequency-dependent stiffness relation between internal forces and dof's

$$
\hat{R}_{j-1, j}(s)=\left[k_{a}+s G_{a}(s)\right]\left[\hat{u}_{j}(s)-\hat{u}_{j-1}(s)\right]
$$

where $\hat{R}_{j-1, j}(s)=\mathcal{L}\left\{R_{j-1, j}(t)\right\}$ and $\hat{u}_{j}(s)=\mathcal{L}\left\{u_{j}(t)\right\}$ are the Laplace transform of the internal forces and of the dof's, respectively.

For viscoelastic links $B$ based on fractional derivatives, an explicit expression of the kernel function $\mathcal{G}_{b}(t)$ is not analytically available. However, the damping function in the Laplace domain $G_{b}(s)$ can easily be calculated simply applying to the fractional derivatives of Eq. (60) the Laplace transform and using its properties. If $\hat{Q}_{j}(s)=\mathcal{L}\left\{Q_{j}(t)\right\}$ is the Laplace transform of the reactions at links $B$, then

$$
\hat{Q}_{j}(s)=k_{b} \frac{1+c\left(T_{r} s\right)^{\gamma}}{1+\left(T_{r} s\right)^{\gamma}} \hat{u}_{j}(s) \equiv\left[k_{b}+s G_{b}(s)\right] \hat{u}_{j}(s)
$$

xviii 
where

$$
G_{b}(s)=\frac{k_{b}}{s} \frac{(c-1)\left(T_{r} s\right)^{\gamma}}{1+\left(T_{r} s\right)^{\gamma}}
$$

The free-motion equations in the Laplace domain can be obtained assembling the mass and the stiffness matrices associated with the structural configuration shown in Fig. 3, resulting in an equilibrium similar to that of Eq. (2)

$$
\left[s^{2} \mathbf{M}+s \mathbf{G}(s)+\mathbf{K}\right] \hat{\mathbf{u}}(s)=\mathbf{0}
$$

where $\mathbf{M}=m \mathbf{I}_{4}, \mathbf{K}=k_{a} \boldsymbol{\Pi}_{a}+k_{b} \boldsymbol{\Pi}_{b}$ and $\mathbf{G}=G_{a}(s) \boldsymbol{\Pi}_{a}+G_{b}(s) \boldsymbol{\Pi}_{b}$, with auxiliary matrices

$$
\boldsymbol{\Pi}_{a}=\left[\begin{array}{rrrr}
2 & -1 & 0 & 0 \\
-1 & 2 & -1 & 0 \\
0 & -1 & 2 & -1 \\
0 & 0 & -1 & 2
\end{array}\right], \quad \Pi_{b}=\mathbf{I}_{4}
$$

Table 4 showns the values of mass $m$, static rigidities $k_{a}, k_{b}$ of the springs and natural frequencies of the undamped system. Notice that the damping matrix $\mathbf{G}(s)$ can be expressed as linear combination of the stiffness and mass matrices in the following form

$$
\mathbf{G}(s)=\frac{G_{a}(s)}{k_{a}} \mathbf{K}+\frac{k_{a} G_{b}(s)-k_{b} G_{a}(s)}{k_{a} m} \mathbf{M}
$$

Under these conditions, the viscoelastic damping matrix $\mathbf{G}(s)$ is said to be proportional, [48], and it can be assured that $\boldsymbol{\Gamma}(s)=\boldsymbol{\Phi}^{T} \mathbf{G}(s) \boldsymbol{\Phi}$ is diagonal. Thus, according to the theoretical results presented in Sec. 3 and after some operations, the non-dimensional function $J_{j}(s)$ is

$$
J_{j}(s)=\frac{\phi_{j}^{T} \mathbf{G}(s) \phi_{j}}{2 m_{j} \omega_{j}}=\frac{G_{a}(s)}{2 k_{a}} \omega_{j}+\frac{k_{a} G_{b}(s)-k_{b} G_{a}(s)}{2 k_{a} m \omega_{j}}
$$

where $\omega_{j}$ is the undamped frequency of the $j$ th mode. Consequently, the functions $X_{j}(s), Y_{j}(s)$ defined in Eqs. (47) can be constructed to compute the complex eigenvalues using the iterative process defined by the sequence of iterates

$$
x_{j}^{(n)}=X_{j}\left(x_{j}^{(n-1)}\right), \quad y_{j}^{(n)}=Y_{j}\left(y_{j}^{(n-1)}\right), \quad 1 \leq j \leq 4
$$

As shown in Theorem 3, the convergence speed depends on the damping level induced in the system by the viscoelastic model. For this reason, in the current example three separated cases corresponding to three different damping levels will be studied: lightly damped (LD), moderately damped (MD) and strongly damped (SD) systems. In order to numerically differentiate these levels, the loss factor peak will be used as damping index. As known [53, 54, 55, 56,57], the loss factor of a frequency-domain constitutive equation is a real-valued and frequency-dependent function defined as the quotient between the imaginary and the real part of the complex stiffness. Here, the complex stiffness associated with the viscoelastic links of the structure shown in Fig. 3 are

$$
\begin{array}{llll}
\hat{k}_{a}(i \omega)= & k_{a}+i \omega G_{a}(i \omega)= & M_{a}(\omega)+i L_{a}(\omega)= & M_{a}(\omega)\left[1+i \eta_{a}(\omega)\right] \\
\hat{k}_{b}(i \omega)= & k_{b}+i \omega G_{b}(i \omega)= & M_{b}(\omega)+i L_{b}(\omega)= & M_{b}(\omega)\left[1+i \eta_{b}(\omega)\right]
\end{array}
$$

where $M_{a}(\omega), M_{b}(\omega)$ are the real part of the complex stiffness or dynamic modulus; $L_{a}(\omega), L_{b}(\omega)$ the imaginary part or loss modulus and finally $\eta_{a}(\omega)=L_{a}(\omega) / M_{a}(\omega), \eta_{b}(\omega)=L_{b}(\omega) / M_{b}(\omega)$ the loss factors. In general, for real solid materials, the loss factor presents one maximum peak [52]. The function takes at the peak a representative value of the induced level of damping $\eta_{m}$ named loss factor peak. For the current viscoelastic links, the loss factor peaks are defined as

$$
\eta_{m A}=\max _{\omega \geq 0} \eta_{a}(\omega), \quad \eta_{m B}=\max _{\omega \geq 0} \eta_{b}(\omega)
$$

Pritz [55] studied the frequency dependence of the loss factor for real solid materials. According to the experimental evidence, the reference gave the following classification by order of $\eta_{m}$ level: 


\begin{tabular}{lcccccc} 
& \multicolumn{3}{c}{ Links type $A$} & & \multicolumn{3}{c}{ Links type $B$} \\
\cline { 2 - 3 } \cline { 6 - 7 } Cases & $\zeta$ & $\eta_{m A}$ & & $c$ & $\gamma$ & $\eta_{m B}$ \\
\hline Lightly Damped & 0.007 & $\mathbf{0 . 0 1 0 0}$ & & 1.135 & 0.20 & $\mathbf{0 . 0 1 0 0}$ \\
Moderately Damped & 0.078 & $\mathbf{0 . 1 0 0 1}$ & & 1.860 & 0.40 & $\mathbf{0 . 1 0 0 3}$ \\
Strongly Damped & 2.000 & $\mathbf{1 . 0 0 1 2}$ & & 94.00 & 0.60 & $\mathbf{1 . 0 0 9 1}$ \\
\hline
\end{tabular}

Table 5: Example 2: Numerical cases and computed loss factor peaks of the viscoelastic links for $\left\{\mu_{l}\right\}_{l=1}^{5}=\{5,10,13,18,40\}$ $\mathrm{rad} / \mathrm{s}, T_{r}=10^{-3} \mathrm{~s}$.

- Stiff structural materials present light damping with low $0.001 \leq \eta_{m} \leq 0.01$

- Moderately damped materials, such as plastics, with medium $0.01 \leq \eta_{m} \leq 0.10$

- Rubbers and rubber-like materials used for vibrations control, induce high damping level and consequently $0.10 \leq \eta_{m} \leq 1.0$

This classification is used in the current example to differentiate the three numerical cases. The relaxation parameters of the Biot's multiexponential model (links $A$ ) are $\mu_{l}=\{5,10,13,18,40\} \mathrm{rad} / \mathrm{s}$. For links $B$, the relaxation time is $T_{r}=10^{-3} \mathrm{~s}$ and the rest of parameters, say $\zeta, c, \gamma$ are chosen so that the computed loss factor peaks given by Eq. (71) take the values: LD is associated with $\eta_{m} \approx 0.01$, MD with $\eta_{m} \approx 0.10$ and SD with $\eta_{m} \approx 1.00$. Table 5 shows the parameters and the calculated loss factor peak values for each link type, which will be used in the following.

The results of the recursive scheme are presented in Table 6. For each mode, the initial value is the undamped eigenvalue $x_{j}^{(0)}=i \omega_{j}$. As before, the iteration sequence continues until the non-dimensional iterative error $\epsilon_{j}^{(n)}$ defined as in Eq. (58) is lower than the prefixed value $\epsilon_{\max }=10^{-12}$. The number of computed iterations $N$ shown in the last column increases with the level of damping and as predicted, lightly damped systems present faster convergence. In Fig. 4 the path traced by the elements of the sequence $\left\{x_{j}^{(n)}\right\}_{n=0}^{N}$ in the complex plane has been drawn for each of the four modes, top figures. In addition, the error versus iteration curves have been plotted, bottom figures. For LD, MD the recursive sequences in the complex plane do not present excessive numerical oscillations around the fixed point (square). However, the path for SD behaves similarly as those of the highly damped cases from Example 1. In this case, the first iterations fluctuate, and the error does not decay linearly, a characteristic of contractive functions. Only when the elements of the sequence are close enough to the fixed point, the rate of decay remains constant.

\section{Conclusions}

In this paper, a new numerical method to compute the eigenvalues of linear viscoelastic structures is developed. The method can be implemented for single or multiple degree of freedom systems with a proportional, or lightly non-proportional, damping matrix. Several transformations in the characteristic equation lead us to find two relevant complex functions to be used in a recursive scheme.

The main theoretical conclusions are presented in three theorems. With the help of fixed point theory, Theorem 1 demonstrates the existence of complex eigenvalues considered as fixed points of the recursive functions. Theorems 2 and 3 analyze the convergence of the recursive sequence by means of the Banach fixed point theorem. It is shown that the convergence speed of the proposed recursive method depends on the level of damping and on the viscoelasticity. It is proved that systems with low-damping together with low viscoelasticity present in general faster convergence. For strongly damped structures the iterative process is slower although the convergence is ensured. 


\begin{tabular}{lrrrr} 
Cases & $j$ & $x_{j}^{(0)}$ & $x_{j}^{(N)}$ & $N$ \\
\hline Lightly Damped & 1 & $9.391 i$ & $-0.0395+9.5017 i$ & 7 \\
& 2 & $13.718 i$ & $-0.0637+13.8311 i$ & 7 \\
& 3 & $17.658 i$ & $-0.0865+17.7910 i$ & 7 \\
\hline Moderately Damped & 4 & $20.292 i$ & $-0.1012+20.4453 i$ & 7 \\
& 1 & $9.391 i$ & $-0.3504+9.7831 i$ & 9 \\
& 2 & $13.718 i$ & $-0.6568+14.3957 i$ & 10 \\
& 3 & $17.658 i$ & $-0.9195+18.7015 i$ & 11 \\
Strongly Damped & 4 & $20.292 i$ & $-1.0804+21.6179 i$ & 11 \\
& 1 & $9.391 i$ & $-12.4924+25.1788 i$ & 41 \\
& 2 & $13.718 i$ & $-13.6958+37.8174 i$ & 56 \\
& 3 & $17.658 i$ & $-14.0601+49.3423 i$ & 74 \\
\hline
\end{tabular}

Table 6: Example 2: Approximated eigenvalues $x_{N}$ after $N$ iterations. Sequence is considered to converge if relative error satisfies $\epsilon_{j}^{(N)}<10^{-12}$.

To illustrate and validate the theoretical results, two numerical examples are analyzed. First, a single degree of freedom viscoelastic system with a Biot damping model is studied. Since the overdamped region is available, the level of damping and the speed of the numerical method can be related. In all the numerical cases considered, the recursive sequence has always converged, even when the system is overdamped. Moreover, this example shows that under certain conditions the method may also be used to obtain nonviscous eigenvalues. As predicted by the theory, the cases associated with low damping systems converged faster, and conversely, high damping systems converged slowly. Second, in order to study multiple degree of freedom systems, a four-mass discrete structure with proportional damping is examined. The damping is introduced through viscoelastic links using again Biot's model with five kernels and a damping model based on the fractional derivatives. With the objective to relate the level of damping with the convergence three cases with different levels are studied. Further research is currently being developed by the authors for the generalization of the method for multiple degrees of freedom systems including a damping matrix whose non-proportionality cannot be neglected.

\section{Appendix A. Proof of properties P1 to P5}

Proof of P1. The complex functions $X(s), Y(s)$ defined in Eqs. (10) can be considered a composition of analytical functions. Hence, $X=\omega_{u}(f \circ J), Y=\omega_{u}(g \circ J)$, where

$$
f(z)=-z+i \sqrt{1-z^{2}}, \quad g(z)=-z-i \sqrt{1-z^{2}}
$$

are analytical functions for any $z \in \mathbb{C}$ except in the set $\{z \in \mathbb{R}:|z| \geq 1\}$. Therefore, from the functions' composition properties, $X(s), Y(s)$ are analytical in the set where $J(s)$ is, except in points of the region $\mathcal{B}=\{z \in \mathbb{R}:|J(z)| \geq 1\}$ where the square root is not analytical.

Proof of P2. Since $X(s), Y(s)$ are analytical and consequently holomorphic in the set $\mathcal{A}$, then

$$
X^{*}(s)=\omega_{u}\left[-J(s)+i \sqrt{1-J^{2}(s)}\right]^{*}=\omega_{u}\left[-J\left(s^{*}\right)+(-i) \sqrt{1-J^{2}\left(s^{*}\right)}\right]=Y\left(s^{*}\right)
$$



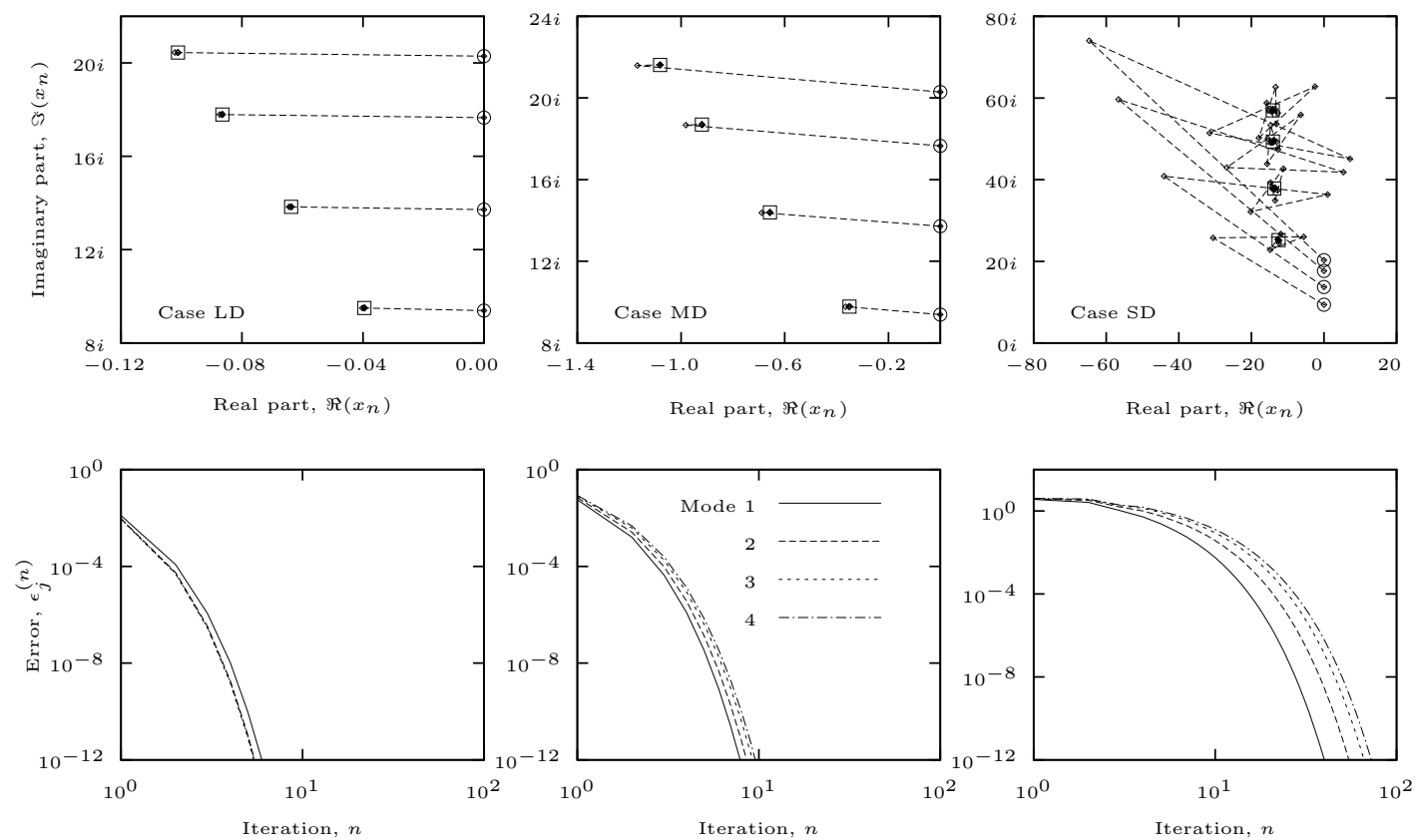

Figure 4: Example 2: Four mode paths in complex plane of iterates' sequence from initial point $\odot$ to limit eigenvalue $\square$ for Lightly, Moderately and Strongly Damped, top figures. Associated error $\epsilon_{j}^{(n)}$, bottom figures.

Proof of P3. Let $z \in \mathcal{A}$ be a fixed point of $X(s)$, then $z=X(z)$. Taking the complex conjugate of both terms and using $\mathrm{P} 2$, it follows that $z^{*}=X^{*}(z)=Y\left(z^{*}\right)$, from where $z^{*}$ is a fixed point of $Y(s)$. It is clear that using the same principle the reciprocal also holds.

Proof of P4. From the hypothesis H3, $J(s) \in \mathcal{C}, \forall s \in \mathcal{C}$. Due to $X=\omega_{u}(f \circ J)$, it follows that $X(\mathcal{C}) \subset \mathcal{C}_{+}$ holds only if $f(\mathcal{C}) \subset \mathcal{C}_{+}$. Taking a complex number $z=x+i y \in \mathcal{C}$, it is necessary to prove that $\Im\{f(z)\}>0$. By the definition of the imaginary part of a complex number

$$
\begin{aligned}
\Im\{f(z)\} & =\frac{f(z)-f^{*}(z)}{2 i}=\frac{1}{2 i}\left(-z+i \sqrt{1-z^{2}}+z^{*}-(-i) \sqrt{1-z^{* 2}}\right) \\
& =-\frac{z-z^{*}}{2 i}+\frac{\sqrt{1-z^{2}}+\sqrt{1-z^{* 2}}}{2 i}=-\Im\{z\}+\Re\left\{\sqrt{1-z^{2}}\right\}
\end{aligned}
$$

Let now $u+i v \in \mathbb{C}$ be the main square root of $1-z^{2}$, then $(u+i v)^{2}=1-z^{2}=1-(x+i y)^{2}$ where $u=\Re\left\{\sqrt{1-z^{2}}\right\} \geq 0$. Let us calculate $u$ by identification of real and imaginary parts in the previous equality

$$
u^{2}-v^{2}=1-x^{2}+y^{2}, \quad u v=-x y
$$

From the expression, an equation in only the variable $u$ may be deduced. Thus, the real part $u$ may be computed as a root of the four-order polynomial $u^{4}-\rho u^{2}-(x y)^{2}=0$, where $\rho=1-x^{2}+y^{2}$. Therefore, cases $x=0, x \neq 0$ must be considered separately.

If $x=0$ then $\rho=1+y^{2}$ and the polynomial equation leads to $u^{2}\left(u^{2}-1-y^{2}\right)=0$, hence, either $u=0$ or $u^{2}=1+y^{2}$. For the first, it is verified from Eq. (A.4) that $-v^{2}=1+y^{2}>0$, a contradiction; consequently $u^{2}=1+y^{2}$. The negative solution is not of interest since $u$ is defined by a main square root. Therefore

$$
u=\Re\left\{\sqrt{1-z^{2}}\right\}=\sqrt{1+y^{2}}>y=\Im\{z\} \Rightarrow \Im\{f(z)\}>0 .
$$


If $x \neq 0$, the solutions of the four-order polynomial can be expressed in the form $u^{2}=\left(\rho \pm \sqrt{\rho^{2}+4(x y)^{2}}\right) / 2$. Since $u^{2}>0$, the solution $\rho-\sqrt{\rho^{2}+4(x y)^{2}}<0$ must be rejected. Now, let us suppose that $u=$ $\Re\left\{\sqrt{1-z^{2}}\right\} \leq y=\Im\{z\}$ in order to arrive to a contradiction

$$
\begin{aligned}
0 \leq u \leq y \quad \Rightarrow \quad u^{2} \leq 2 y^{2} \Rightarrow \rho+\sqrt{\rho^{2}+4(x y)^{2}} \leq 2 y^{2} & \Rightarrow \\
\sqrt{\rho^{2}+4(x y)^{2}} \leq 2 y^{2}-\rho \Rightarrow \rho^{2}+4(x y)^{2} \leq 4 y^{2}-4 y^{2} \rho+\rho^{2} & \Rightarrow \\
x^{2} \leq y^{2}-\rho=y^{2}-1+x^{2}-y^{2} & \Rightarrow 0 \leq-1
\end{aligned}
$$

The last inequality is the contradiction, for this reason necessarily $\Im\{f(z)\}=u-y>0$ also for $x \neq 0$. Therefore, $\Im\{X(s)\}>0, \forall s \in \mathcal{C}$ that is equivalent to $X(\mathcal{C}) \subset \mathcal{C}_{+}$.

Now, if $s \in \mathcal{C}$, then $X\left(s^{*}\right) \in \mathcal{C}_{+}$and $\Im\left\{X\left(s^{*}\right)\right\}>0$. Therefore $\Im\left\{X^{*}\left(s^{*}\right)\right\}=\Im\{Y(s)\}<0$ that proves the property for the function $Y(s)$.

Proof of P5. Let us consider an element $x \in \mathcal{B} \subset \mathbb{R}$. From the definition of the set $\mathcal{B},|J(x)| \geq 1$ is verified and from the main hypothesis of the propery $J(\mathbb{R}) \subset \mathbb{R}$, therefore, $z=J(x) \in \mathbb{R}$. Thus, the number $1-z^{2} \leq 0$ and $\sqrt{1-z^{2}}=i \sqrt{\left|1-z^{2}\right|}$ is pure imaginary. Hence, the value of the function is $f(z)=-z-\sqrt{\left|1-z^{2}\right|} \in \mathbb{R}$ so that $X(\mathcal{B}) \subset \mathbb{R}$.

Now, let us consider a number $x \in \mathbb{R} \backslash \mathcal{B}$ and by definition $|J(x)|<1$ and $1-z^{2}>0$. Consequently, $\Im\{f(z)\}=\sqrt{1-z^{2}}>0$ whence $\Im\{X(x)\}>0$ or, in set inclusion notation, $X(\mathbb{R} \backslash \mathcal{B}) \subset \mathcal{C}_{+}$. For statements $Y(\mathcal{B}) \subset \mathbb{R}$ and $Y(\mathbb{R} \backslash \mathcal{B}) \subset \mathcal{C}_{-}$, the same procedure as that of $X(s)$ can be used.

\section{Acknowledgments}

The authors gratefully acknowledge the support of the Polytechnic University of Valencia under programs PAID 02-11-1828 and 05-10-2674 and of the National Science and Research Council of Canada.

\section{References}

[1] M. Biot, Variational Principles in Irreversible Thermodynamics with Application to Viscoelasticity, Physical Review 97 (6) (1955) 1463-1469.

[2] R. Bagley, P. Torvik, A Theoretical Basis for the Application of Fractional Calculus to Viscoelasticity, Journal of Rheology 27 (3) (1983) 201-210.

[3] R. Bagley, P. Torvik, Fractional Calculus - A Different Approach to the Analysis of Viscoelastically Damped Structures, AIAA Journal 21 (5) (1983) 741-748.

[4] Y. A. Rossikhin, M. V. Shitikova, Application of Fractional Calculus for Dynamic Problems of Solid Mechanics: Novel Trends and Recent Results, Applied Mechanics Reviews 63 (1) (2010) 010801(1)-010801(52).

[5] S. Ray, R. Bera, Analytical solution of the Bagley Torvik equation by Adomian decomposition method, Applied Mathematics and Computation 168 (1) (2005) 398-410.

[6] S. S. Ray, K. S. Chaudhuri, R. K. Bera, Analytical approximate solution of nonlinear dynamic system containing fractional derivative by modified decomposition method, Applied Mathematics and Computation 182 (1) (2006) 544-552.

[7] S. S. Ray, On Haar wavelet operational matrix of general order and its application for the numerical solution of fractional Bagley Torvik equation, Applied Mathematics and Computation 218 (9) (2012) 5239-5248.

[8] D. Golla, P. Hughes, Dynamics of Viscoelastic Structures - A Time-domain, Finite-element Formulation, Journal of Applied Mechanics, Transactions ASME 52 (4) (1985) 897-906.

[9] K. J. Buhariwala, Dynamic response of viscoelastic fibre reinforced composite materials, Master's thesis, Institute for Aerospace Studies. University of Toronto. (1982).

[10] H. J. Buhariwala, K.J., Dynamics of Viscoelastic Structures, AIAA Journal 26 (2) (1988) 220-227.

[11] D. McTavish, P. Hughes, Modeling of linear viscoelastic space structures, Journal of Vibration and Acoustics-Transactions of the ASME 115 (1) (1993) 103-110.

[12] G. Lesieutre, D. Mingori, Finite-Element Modeling of Frequency-dependent Material Damping Using Augmenting Thermodynamic Fields, Journal of Guidance Control and Dynamics 13 (6) (1990) 1040-1050.

[13] S. Adhikari, Energy dissipation in vibrating structures, Master's thesis, Cambridge University Engineering Department, Cambridge, UK, first Year Report (May 1998). 
[14] S. Adhikari, J. Woodhouse, Identification Of Damping: PART 2, Non-Viscous Damping, Journal of Sound and Vibration 243 (1) (2001) 63-88.

[15] M. Medjden, N. eddine Tatar, Asymptotic behavior for a viscoelastic problem with not necessarily decreasing kernel, Applied Mathematics and Computation 167 (2) (2005) 1221 - 1235.

[16] N.-e. Tatar, Uniform decay in viscoelasticity for kernels with small non-decreasingness zones, Applied Mathematics and Computation 218 (15) (2012) 7939-7946.

[17] W. H. Yang, A method for eigenvalues of sparse $\lambda$-matrices, International Journal for Numerical Methods in Engineering 19 (6) (1983) 943-948.

[18] K. Singh, Y. Ram, Transcendental eigenvalue problem and its applications, AIAA Journal 40 (7) (2002) $1402-1407$.

[19] F. Williams, D. Kennedy, Reliable Use of Determinants to Solve Non-linear Structural Eigenvalue Problems Efficiently, International Journal for Numerical Methods in Engineering 26 (8) (1988) 1825-1841.

[20] E. M. Daya, M. Potier-Ferry, A numerical method for nonlinear eigenvalue problems application to vibrations of viscoelastic structures, Computers \& Structures 79 (5) (2001) $533-541$.

[21] L. Duigou, E. M. Daya, M. Potier-Ferry, Iterative algorithms for non-linear eigenvalue problems. application to vibrations of viscoelastic shells, Computer Methods in Applied Mechanics and Engineering 192 (11-12) (2003) 1323 - 1335.

[22] H. Voss, An arnoldi method for nonlinear eigenvalue problems, BIT Numerical Mathematics 44 (2004) 387-401.

[23] H. Voss, A Jacobi-Davidson method for nonlinear and nonsymmetric eigenproblems, Computers \& Structures 85 (17-18) (2007) 1284-1292.

[24] M. Abdel-Aziz, S. El-Sayed, Sensitivity analysis of the largest dependent eigenvalue functions of eigensystems, Applied Mathematics and Computation 100 (2-3) (1999) 103-110.

[25] A. Muravyov, S. Hutton, Closed-form solutions and the eigenvalue problem for vibration of discrete viscoelastic systems, Journal of Applied Mechanics-Transactions of The ASME 64 (3) (1997) 684-691.

[26] A. Muravyov, Forced vibration responses of viscoelastic structure, Journal of Sound and Vibration 218 (5) (1998) $892-907$.

[27] S. Menon, J. Tang, A state-space approach for the dynamic analysis of viscoelastic systems, Computers \& Structures $82(15-16)(2004) 1123-1130$.

[28] S. Adhikari, B. Pascual, Eigenvalues of linear viscoelastic systems, Journal of Sound and Vibration 325 (4-5) (2009) $1000-1011$.

[29] S. Adhikari, B. Pascual, Iterative methods for eigenvalues of viscoelastic systems, Journal of Vibration and Acoustics 133 (2) (2011) 021002.

[30] M. Friswell, S. Adhikari, Y. Lei, Vibration analysis of beams with non-local foundations using the finite element method, International Journal for Numerical Methods in Engineering 71 (2007) 1365-1386.

[31] S. Adhikari, M. I. Friswell, Eigenderivative analysis of asymmetric non-conservative systems, International Journal for Numerical Methods in Engineering 51 (6) (2001) 709-733.

[32] S. Adhikari, Derivative of Eigensolutions of Nonviscously Damped Linear Systems, AIAA Journal 40 (10) (2002) 20612069.

[33] F. Cortés, M. J. Elejebarrieta, Computational methods for complex eigenproblems in finite element analysis of structural systems with viscoelastic damping treatments, Computer Methods Appl. Mech. Engineering 195 (44-47) (2006) 6448-6462.

[34] F. Cortés, M. J. Elejebarrieta, An approximate numerical method for the complex eigenproblem in systems characterised by a structural damping matrix , Journal of Sound and Vibration 296 (1-2) (2006) 166-182.

[35] M. Martinez-Agirre, M. J. Elejabarrieta, Higher order eigensensitivities-based numerical method for the harmonic analysis of viscoelastically damped structures, International Journal for Numerical Methods in Engineering 88 (12) (2011) 12801296.

[36] M. Lázaro, J. L. Pérez-Aparicio, Parametric solutions of the eigenvalue problem for single degree-of-freedom viscoelastic systems, AIAA Journal. 0 (2012) i-xvii, submited Manuscript ID 2012-03-J051941.

[37] M. Lázaro, J. L. Pérez-Aparicio, Multiparametric computation of eigenvalues for linear viscoelastic structures, Computers \& Structures 0 (2012) i-xvii, submited Manuscript ID CAS-D-12-00383.

[38] P. Muller, Are the eigensolutions of a l-d.o.f. system with viscoelastic damping oscillatory or not?, Journal of Sound and Vibration 285 (1-2) (2005) 501-509.

[39] S. Adhikari, Dynamics of Non-viscously Damped Linear Systems, Journal of Engineering Mechanics 128 (3) (2002) 328339.

[40] K. Goebel, T. Sekowski, A. Stachura, Uniform convexity of the hyperbolic metric and fixed point of holomorphic mappings in the hilbert ball, Nonlinear Analysis: Theory, Methods \& Applications 4 (5) (1980) 1011-1021.

[41] S. Reich, Approximating fixed points of holomorphic mappings, Math. Japon. 37 (1992) 457-459.

[42] S. Banach, Sur les opérations dans les ensembles abstraits et leurs applications aux équations intégrales, Fundamenta Mathematicae 3 (1922) 133-181.

[43] W. A. Kirk, B. Sims, Handbook of Metric Fixed Point Theory, Kluwer Academic Publishers, 2001.

[44] R. M. McLeod, Mean value theorems for vector valued functions, Proceedings of the Edinburgh Mathematical Society (Series 2) 14 (03) (1965) 197-209.

[45] A. M. Ostrowski, Solutions of Equations in Euclidean and Banach Spaces, Academic Press, 1973.

[46] S. Adhikari, J. Woodhouse, Quantification of non-viscous damping in discrete linear systems, Journal of Sound and Vibration 260 (3) (2003) 499-518.

[47] E. Schroeder, Über unedlich viele algorithmen zur auflösung der gleichungen, Mathematische Annalen 2 (1870) $317-365$.

[48] S. Adhikari, Classical normal modes in non-viscously damped linear systems, AIAA Journal 39 (5) (2001) 978-980.

[49] J. Woodhouse, Linear Damping Models For Structural Vibration, Journal of Sound and Vibration 215 (3) (1998) $547-569$.

[50] A. Muravyov, S. Hutton, Free vibration response characteristics of a simple elasto-hereditary system, Journal of Vibration 
and Acoustics-Transactions of the ASME 120 (2) (1998) 628-632.

[51] S. Adhikari, Qualitative dynamic characteristics of a non-viscously damped oscillator, Proceedings of the Royal Society A-Mathematical Physical and Engineering Sciences 461 (2059) (2005) 2269-2288.

[52] T. Pritz, Analysis of four-parameter fractional derivative model of real solid materials, Journal of Sound and Vibration 195 (1) (1996) 103-115.

[53] A. Nashif, D. Jones, J. Henderson, Vibration Damping, John Wiley and Sons, 1985.

[54] D. I. Jones, Handbook of viscoelastic vibration damping, John Wiley \& Sons, 2001.

[55] T. Pritz, Frequency dependences of complex moduli and complex Posson's ratio of real solid materials, Journal of Sound and Vibration 214 (1) (1998) 83-104.

[56] T. Pritz, Loss factor peak of viscoelastic materials: Magnitude to width relations, Journal of Sound and Vibration 246 (2) (2001) 265-280

[57] J. de Espindola, J. Neto, E. Lopes, A generalised fractional derivative approach to viscoelastic material properties measurement, Applied Mathematics and Computation 164 (2) (2005) 493-506. 